University of Nebraska - Lincoln

DigitalCommons@University of Nebraska - Lincoln

Timothy J. Gay Publications

Research Papers in Physics and Astronomy

2009

\title{
Use of partial-wave decomposition to identify resonant interference effects in the photoionization-excitation of argon
}

Timothy J. Gay

University of Nebraska - Lincoln, tgay1@unl.edu

Chris H. Greene

University of Colorado at Boulder, chgreene@purdue.edu

J. R. Machacek

University of Nebraska-Lincoln

K. W. McLaughlin

Loras College

H. W. van der Hart

Queen's University - Belfast

See next page for additional authors

Follow this and additional works at: https://digitalcommons.unl.edu/physicsgay

Part of the Physics Commons

Gay, Timothy J.; Greene, Chris H.; Machacek, J. R.; McLaughlin, K. W.; van der Hart, H. W.; Yenen, Orhan; and Jaecks, Duane H., "Use of partial-wave decomposition to identify resonant interference effects in the photoionization-excitation of argon" (2009). Timothy J. Gay Publications. 60.

https://digitalcommons.unl.edu/physicsgay/60

This Article is brought to you for free and open access by the Research Papers in Physics and Astronomy at DigitalCommons@University of Nebraska - Lincoln. It has been accepted for inclusion in Timothy J. Gay Publications by an authorized administrator of DigitalCommons@University of Nebraska - Lincoln. 


\section{Authors}

Timothy J. Gay, Chris H. Greene, J. R. Machacek, K. W. McLaughlin, H. W. van der Hart, Orhan Yenen, and Duane H. Jaecks 


\title{
Use of partial-wave decomposition to identify resonant interference effects in the photoionization-excitation of argon
}

\author{
T J Gay ${ }^{1}$, Chris H Greene ${ }^{2}$, J R Machacek ${ }^{1}$, K W McLaughlin ${ }^{3}$, \\ H W van der Hart ${ }^{4}$, O Yenen ${ }^{1}$ and D H Jaecks ${ }^{1}$ \\ ${ }^{1}$ Behlen Laboratory of Physics, University of Nebraska, Lincoln, NB 68588, USA \\ ${ }^{2}$ Department of Physics and JILA, University of Colorado, Boulder, CO 80309, USA \\ ${ }^{3}$ Department of Physics and Engineering, Loras College, Dubuque, IA 52001, USA \\ ${ }^{4}$ Centre for Theoretical Atomic, Molecular and Optical Physics, Queen's University Belfast, \\ Belfast BT7 1NN, UK \\ E-mail: tgay1@unl.edu
}

Received 25 May 2008, in final form 4 July 2008

Published 3 February 2009

Online at stacks.iop.org/JPhysB/42/044008

\begin{abstract}
We have studied simultaneous photoionization and excitation of Ar in the range of incident photon energies between 36.00 and $36.36 \mathrm{eV}$, where the resonant production of doubly excited neutral Ar states imbedded in the ionization continuum is dominant. By measuring the relative Stokes parameters of the fluorescence from residual $\mathrm{Ar}^{+*}\left(3 \mathrm{p}^{4}\left[{ }^{3} \mathrm{P}\right] 4 \mathrm{p}\right)$ ions $\left({ }^{2} \mathrm{P}_{1 / 2}, 465.8 \mathrm{~nm}\right.$ transition; ${ }^{2} \mathrm{P}_{3 / 2}, 476.5 \mathrm{~nm} ;{ }^{2} \mathrm{D}_{3 / 2}, 472.7 \mathrm{~nm} ;{ }^{2} \mathrm{D}_{5 / 2}, 488.0 \mathrm{~nm} ;{ }^{4} \mathrm{P}_{5 / 2}, 480.6 \mathrm{~nm} ;{ }^{4} \mathrm{D}_{5 / 2}$, $514.5 \mathrm{~nm}$ ) we demonstrate a technique for determining individual partial-wave cross sections in photoionizing collisions. This procedure is shown to be important in sorting out competing dynamical ionization mechanisms, particularly with regard to resonant production of intermediate doubly excited autoionizing states. Comparison with theoretical photoionization cross sections demonstrates that spin-orbit coupling between different states of Ar II needs to be accounted for in the calculations.
\end{abstract}

(Some figures in this article are in colour only in the electronic version)

\section{Introduction}

An important goal in the study of multi-electron dynamics during photoionization is to delineate the specific mechanisms that govern energy and angular momentum partitioning between excited and outgoing electrons. Argon provides a convenient atomic laboratory for new experimental approaches to achieve this goal because it has no hyperfine structure, and its fine structure is easily resolvable by fluorescence measurements. As the intensity of synchrotron radiation sources has improved through a second and third generation, the detail with which photoionization processes can be studied has steadily improved. Initial studies involving total absorption cross sections [1, 2] have been superseded by experiments in which final residual ion states are identified, either through photoelectron energy analysis [3-6] or by fluorescence detection [7-12].
Photoionization that is accompanied by electronic excitation of the residual ion represents a rigorous test of photoionization theory for multi-electron atoms because it cannot occur in the independent electron picture. This is particularly true in the energy region just above the excitation thresholds for the various possible ionic 'satellite' states, where the outgoing electron leaves the collision volume slowly, and ionization occurs primarily through the resonant production of doubly excited neutral states which subsequently autoionize. An energy-level diagram [13] of the Ar II system above $35.0 \mathrm{eV}$ (from the ground state of Ar I) is shown in figure 1. The Ar II states are Rydberg series limits of the doubly excited autoionizing resonances. This energy regime can yield extremely complicated spectra because of the density in energy space of these doubly excited resonant states. Isolation of specific dynamical mechanisms for a given excitation/ionization channel can thus be very difficult 


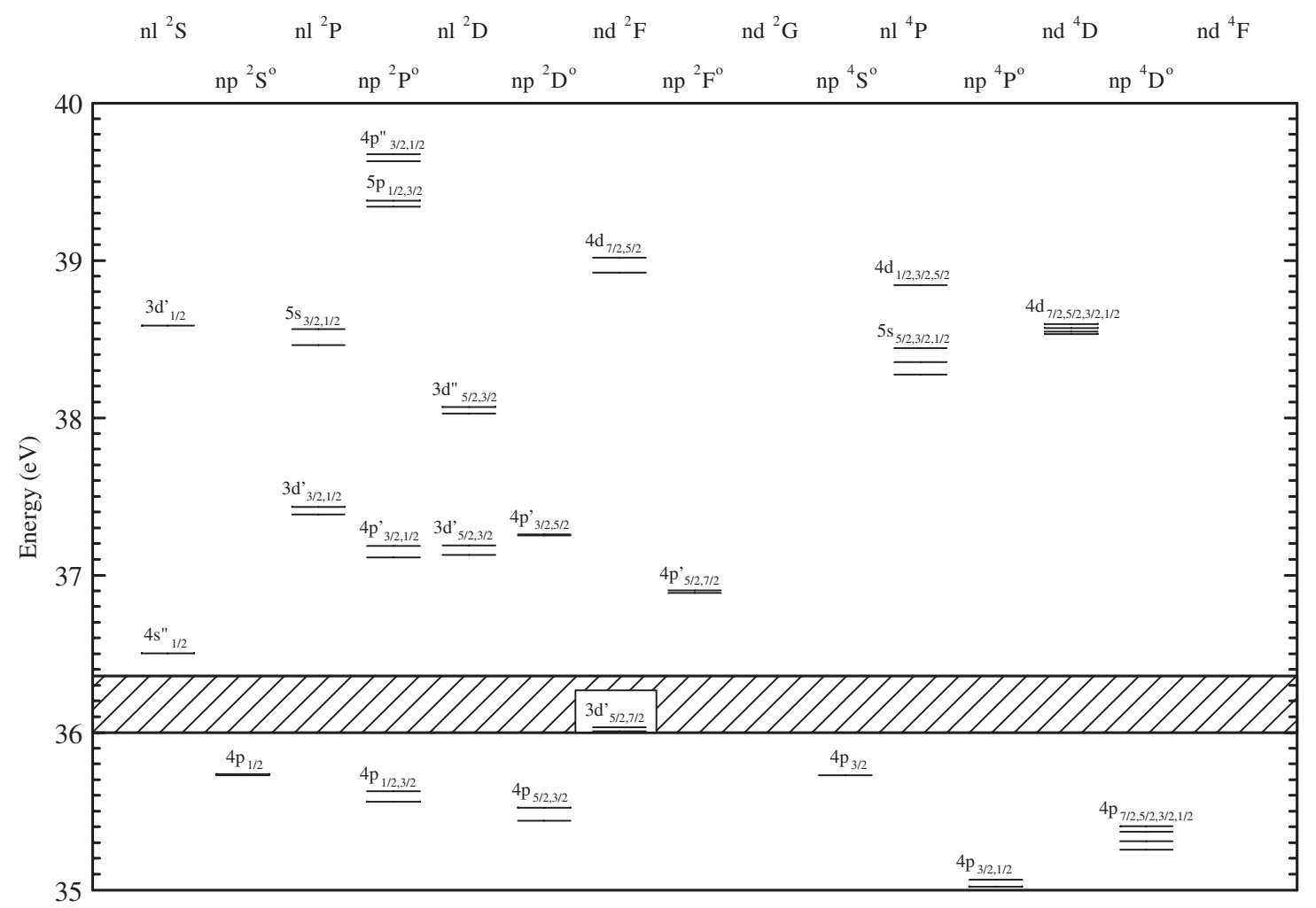

Figure 1. Energy level diagram of Ar II. The state energy values, relative to the ground state of Ar I, are taken from [13]. The configurations indicated with no primes correspond to $3 \mathrm{p}^{4}\left[{ }^{3} \mathrm{P}\right]$ cores; those with one prime have $3 \mathrm{p}^{4}\left[{ }^{1} \mathrm{D}\right]$ cores; those with two primes have $3 \mathrm{p}^{4}\left[{ }^{1} \mathrm{~S}\right]$ cores. The energy range of data discussed in this paper is in the shaded area.

under these circumstances. In this context, fluorescence measurements as a means of identifying the specific ionic state in the final photoionization channel have the important advantages of relatively high energy resolution and an independence of their detection efficiency on the emitted electron energy, making them particularly suitable for the study of spectral features near a given reaction threshold. One obvious limitation of this method is the possibility of fluorescent cascading from higher excited states of the ion. This possibility can be eliminated by keeping the incident photon energy below the first cascading threshold.

As third generation synchrotrons have come on line, the possibility of making such measurements in this energy range with resolution of a few $\mathrm{meV}$ - comparable to absorption measurements [1, 2]-has been realized. More recently, polarization analysis of the fluorescence has been shown to have the ability to yield even finer detail about the final state of the photoionization reaction by identifying outgoing electron partial waves and/or the alignment and orientation of the residual ion [8-12].

In this paper, following earlier brief reports [11, 12], we discuss the technique for extracting electron partial-wave cross sections from ionic fluorescence measurements. We then present data on partial-wave cross sections for six transitions in Ar II, concentrating on an energy range with particularly strong, relatively isolated resonances. The six transitions all involve photo-production of residual ionic states with a $\left(3 s^{2} 3 p^{4} 4 p\right)$ configuration in which the $3 p$ electrons are coupled into a ${ }^{3} \mathrm{P}$ core:

$$
\begin{aligned}
\gamma+ & \operatorname{Ar}\left(3 \mathrm{p}^{6}\right) \rightarrow \operatorname{Ar}^{+}\left(3 \mathrm{p}^{4}\left[{ }^{3} \mathrm{P}\right] 4 \mathrm{p}\right)^{2} \mathrm{P}_{1 / 2}^{\circ}+\mathrm{e}^{-} \\
\rightarrow & \operatorname{Ar}^{+}\left(3 \mathrm{p}^{4}\left[{ }^{3} \mathrm{P}\right] 4 \mathrm{~s}\right)^{2} \mathrm{P}_{3 / 2}+\gamma(465.8 \mathrm{~nm}) \\
\rightarrow & \operatorname{Ar}^{+}\left(3 \mathrm{p}^{4}\left[{ }^{3} \mathrm{P}\right] 4 \mathrm{p}\right)^{2} \mathrm{P}_{3 / 2}^{\circ}+\mathrm{e}^{-} \\
\rightarrow & \operatorname{Ar}^{+}\left(3 \mathrm{p}^{4}\left[{ }^{3} \mathrm{P}\right] 4 \mathrm{~s}\right)^{2} \mathrm{P}_{1 / 2}+\gamma(476.5 \mathrm{~nm}) \\
\rightarrow & \operatorname{Ar}^{+}\left(3 \mathrm{p}^{4}\left[{ }^{3} \mathrm{P}\right] 4 \mathrm{p}\right)^{2} \mathrm{D}_{3 / 2}^{\circ}+\mathrm{e}^{-} \\
& \rightarrow \operatorname{Ar}^{+}\left(3 \mathrm{p}^{4}\left[{ }^{3} \mathrm{P}\right] 4 \mathrm{~s}\right)^{2} \mathrm{P}_{3 / 2}+\gamma(472.7 \mathrm{~nm}) \\
\rightarrow & \operatorname{Ar}^{+}\left(3 \mathrm{p}^{4}\left[{ }^{3} \mathrm{P}\right] 4 \mathrm{p}\right)^{2} \mathrm{D}_{5 / 2}^{\circ}+\mathrm{e}^{-} \\
& \rightarrow \operatorname{Ar}^{+}\left(3 \mathrm{p}^{4}\left[{ }^{3} \mathrm{P}\right] 4 \mathrm{~s}\right)^{2} \mathrm{P}_{3 / 2}+\gamma(488.0 \mathrm{~nm}) \\
\rightarrow & \operatorname{Ar}^{+}\left(3 \mathrm{p}^{4}\left[{ }^{3} \mathrm{P}\right] 4 \mathrm{p}\right)^{4} \mathrm{P}_{5 / 2}^{\circ}+\mathrm{e}^{-} \\
& \rightarrow \operatorname{Ar}^{+}\left(3 \mathrm{p}^{4}\left[{ }^{3} \mathrm{P}\right] 4 \mathrm{~s}\right){ }^{4} \mathrm{P}_{5 / 2}+\gamma(480.6 \mathrm{~nm}) \\
\rightarrow & \operatorname{Ar}^{+}\left(3 \mathrm{p}^{4}\left[{ }^{3} \mathrm{P}\right] 4 \mathrm{p}\right)^{4} \mathrm{D}_{5 / 2}^{\circ}+\mathrm{e}^{-} \\
& \rightarrow \operatorname{Ar}^{+}\left(3 \mathrm{p}^{4}\left[{ }^{3} \mathrm{P}\right] 4 \mathrm{~s}\right)^{2} \mathrm{P}_{3 / 2}+\gamma(514.5 \mathrm{~nm})
\end{aligned}
$$

Figure 1 shows that the lowest-lying Ar II state that can cascade into one of the upper states of interest is the $\left(3 p^{4}\left[{ }^{1} \mathrm{~S}\right] 4 \mathrm{~s}\right){ }^{2} \mathrm{~S}_{1 / 2}$ state at $36.504 \mathrm{eV}$. Thus none of our results below this energy are affected by cascading. With the exception of the ${ }^{4} \mathrm{P}_{5 / 2}$ level, none of the $3 \mathrm{p}^{4}\left[{ }^{3} \mathrm{P}\right] 4 \mathrm{p}$ states are well described in an $L S$ basis. The ${ }^{4} \mathrm{P}_{5 / 2}$ ' state is a very pure quartet, but has a $3 \%$ admixture of $\left[{ }^{3} \mathrm{P}\right] 4 \mathrm{p}{ }^{4} \mathrm{D}[14]$. 


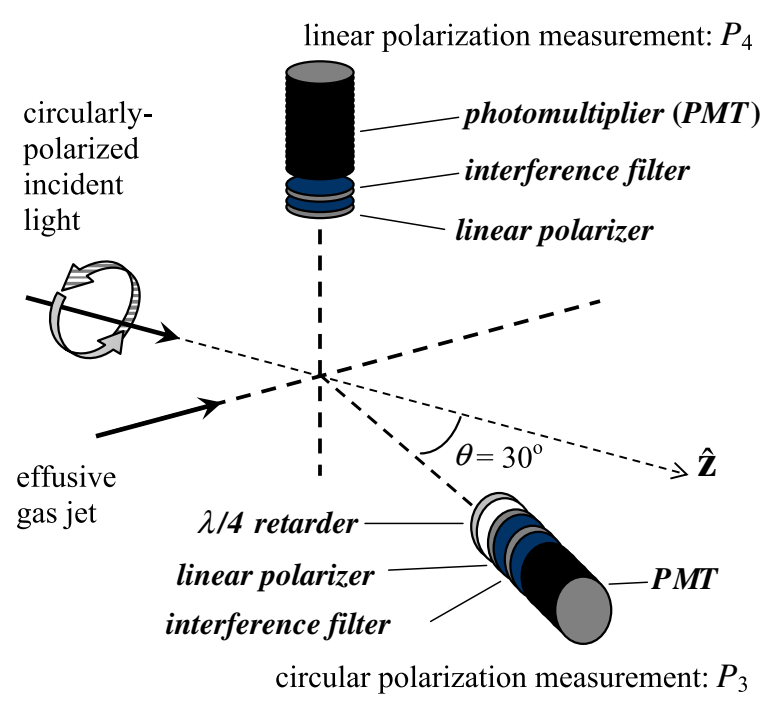

Figure 2. Experimental schematic of the interaction region within the vacuum chamber.

We emphasize in this paper the importance of extracting individual partial waves for the interpretation of a variety of resonant spectral features, particularly with regard to the dynamic processes responsible for them. Among other things, these data provide the first unambiguous evidence in this system for interference between two resonant production mechanisms. In earlier work [12], we showed how electron partial-wave cross sections for reaction (2) were affected by non-specified magnetic interactions, in that they did not display statistical population ratios expected in a pure $L S$ production scheme. (Similar non-statistical distributions of residual Ar II fine-structure components (integrated over partial waves) have been observed in $\mathrm{Ar}$ and $\mathrm{Ne}$ photoelectron spectra $[5,6]$.) Here, we extend this picture to include interference phenomena and discuss the possible roles played by continuum and ionic spin-orbit couplings in terms of their effect on the observed spectra.

\section{Experiment}

Our measurements were performed at beamline 10.0.1.2 of the Advanced Light Source (ALS), Lawrence Berkeley National Laboratory. A spherical-grating monochromator was used to select ionizing photon energies between 36.0 and $36.36 \mathrm{eV}$, with resolutions between 5 and $10 \mathrm{meV}$, controlled by entrance and exit slits on the monochromator. This radiation had a linear polarization in the horizontal plane of the storage ring that was $>99 \%$. Apertures and mirrors following the exit slit from the monochromator produced a beam of roughly circular cross section $0.5 \mathrm{~mm}$ in diameter that intersected the effusive Ar target (figure 2).

Prior to entering the interaction region, the linearly polarized synchrotron radiation traversed a four-reflector retarder which rendered it circularly polarized. The retarder design was based on earlier prototypes $[15,16]$. We measured the circular polarization with a Au mirror analyser to be greater than $99 \%$ at all incident beam energies. As illustrated in figure 2, the photon-gas collision region was observed by optical detection systems at polar angles of $30^{\circ}$ and $90^{\circ}$ relative to the incident photon propagation axis. These optical systems comprised BK7 glass f/1.9 lenses one focal length away from the interaction region (located by the intersection of the axes of the ionizing photon beam and the effusive gas jet, whose needle tip was $2 \mathrm{~mm}$ from this intersection), a quarter-wave retarder (Meadowlark achromat with retardance between 0.253 and 0.244 waves over the wavelength range of interest) for the $30^{\circ}$ system, linear polarizers (Sterling Optics $105 \mathrm{MB}$ ) that could be rotated in situ, interference filters to select the fluorescent transition under study, and lenses to refocus the collimated light onto the bi-alkali photocathodes of the photon-counting photomultiplier tubes. Ti cones whose axes were coincident with the centre of the interaction region were used to shield the insulating surfaces of the first focusing lens in these optical trains. The front ends of these cones were $2.7 \mathrm{~cm}$ from the interaction region. The interference filters used to isolate transitions (1)-(6) had FWHM band-pass widths of $\sim 0.4 \mathrm{~nm}$. A two-layer magnetic shield with one layer internal to the vacuum chamber and another external attenuated the ambient magnetic field in the volume of the interaction region to less than $0.5 \mu \mathrm{T}$.

At each incident photon energy, detector counts were accumulated for a fixed time period and normalized to the digitized signal from the downstream photodiode after a darkcount rate had been subtracted from the raw signal. Our relative intensity data are not corrected for diode quantum efficiency over the energy range of our measurements; from 36.0 to $36.4 \mathrm{eV}$, this efficiency changes by less than $1 \%$. A gasout background with the incident photon beam present never differed statistically from the dark-count rates for the PMTs. There were no systematic shifts between the normalized count rates for data acquisition between ALS storage ring refills.

\section{Theory}

\subsection{Extraction of partial-wave cross sections}

The direct results from our measurements are the polarizations and relative total intensities of selected, fine structure $(J)$ resolved fluorescent transitions. From such data, one means of obtaining the probabilities for the production of specific photoelectron partial waves with total angular momentum $j_{e}$ involves an application of the Fano-Macek [17] formalism, later extended by Greene and Zare [18], whereby one defines parameters that represent a measure of the magnetic sub-state distribution of an atomic state $\psi$. The orientation parameter

$$
\begin{aligned}
O_{0} & \equiv \frac{\left\langle J_{z}\right\rangle}{J(J+1)}=\frac{\sum_{M_{J}} M_{J}\left|\left\langle J M_{J} \mid \psi\right\rangle\right|^{2}}{J(J+1)} \\
& =\frac{2 P_{3}\left[P_{4} \cos ^{2}(\theta)-1\right]}{h_{1}\left(3-P_{4}\right) \cos (\theta)}
\end{aligned}
$$

is proportional to the magnetic dipole moment of the fluorescing atomic state along the incident photon axis $(\hat{z})$, where $\theta$ is the polar angle relative to $\hat{z}$ (see figure 2 ). The 
alignment parameter

$$
\begin{aligned}
A_{0} & \equiv \frac{\left\langle 3 J_{z}^{2}-J^{2}\right\rangle}{J(J+1)}=\frac{\sum_{M_{J}}\left[3 M_{J}^{2}-J(J+1)\right]\left|\left\langle J M_{J} \mid \psi\right\rangle\right|^{2}}{J(J+1)} \\
& =\frac{4 P_{4}}{h_{2}\left(3-P_{4}\right)}
\end{aligned}
$$

is proportional to the zeroth component of the electric quadrupole tensor along this axis. The orientation parameter indicates whether the atomic state has an angular momentum projection parallel or anti-parallel to this symmetry direction, while the alignment parameter indicates whether its charge cloud is prolate or oblate along the same axis. The circular polarization $P_{3}$ is measured at an angle $\theta=30^{\circ}$ to the incident photon momentum (see figure 2), while the linear polarization $P_{4}$ is measured perpendicular to this momentum. The influence of the total angular momenta of the upper $J$ and lower state $J_{f}$ for the fluorescence is contained in the $h_{k}$ term

$$
h_{k} \equiv(-1)^{J-J_{f}} \frac{\left\{\begin{array}{ccc}
J & J & k \\
1 & 1 & J_{f}
\end{array}\right\}}{\left\{\begin{array}{lll}
J & J & k \\
1 & 1 & J
\end{array}\right\}} .
$$

To make the connection between fluorescent polarization and the photoelectron partial-wave probabilities, we denote the final state with a specific photoelectron partial wave by $\left|\psi_{j_{e}}\right\rangle$, which is produced from the initial state $\left|J_{0} M_{J_{0}}\right\rangle$ through the action of the photon absorption operator: $\left|\psi_{j_{e}}\right\rangle=D_{q}^{k}\left|J_{0} M_{J_{0}}\right\rangle$. Projecting onto basis states for the total angular momentum $\left|J_{t} M_{J_{t}}\right\rangle$

$$
\left|\psi_{j_{e}}\right\rangle=\sum_{J_{t}, M_{J_{t}}}\left|J_{t} M_{J_{t}}\right\rangle\left\langle J_{t} M_{J_{t}}\left|D_{q}^{k}\right| J_{0} M_{J_{0}}\right\rangle
$$

allows the use of the Wigner-Eckart theorem

$$
\left\langle J_{t} M_{J_{t}}\left|D_{q}^{k}\right| J_{0} M_{J_{0}}\right\rangle=\left\langle J_{0} M_{J_{0}} k q \mid J_{t} M_{J_{t}}\right\rangle\left\langle J_{t}\left\|D^{k}\right\| J_{0}\right\rangle
$$

to rewrite the final state for a specific photoelectron partial wave

$$
\left|\psi_{j_{e}}\right\rangle=\sum_{J_{t}, M_{J_{t}}}\left|J_{t} M_{J_{t}}\right\rangle\left\langle J_{0} M_{J_{0}} k q \mid J_{t} M_{J_{t}}\right\rangle\left\langle J_{t}\left\|D^{k}\right\| J_{0}\right\rangle .
$$

Within the dipole approximation $k=1$. For circularly polarized ionizing radiation of right-handed helicity, $q=+1$, and with the atom initially in its ground state with $J_{0}=0$ and $M_{J_{0}}=0$ the Clebsch-Gordan coefficient $\left\langle J_{0} M_{J_{0}} k q \mid J_{t} M_{J_{t}}\right\rangle$ vanishes unless $J_{t}=1$ and $M_{J_{t}}=+1$. Therefore, the final state for a specific photoelectron partial wave reduces to

$$
\left|\psi_{j_{e}}\right\rangle=\left|J_{t}=1, M_{J_{t}}=+1\right\rangle\left\langle J_{t}\left\|D^{k}\right\| J_{0}\right\rangle .
$$

We can decouple the total final-state angular momentum $\overrightarrow{\mathbf{J}}_{\mathbf{t}}=\overrightarrow{\mathbf{J}}+\overrightarrow{\mathbf{j}}_{\mathbf{e}}$ into that for the ion $\overrightarrow{\mathbf{J}}$ and the photoelectron $\overrightarrow{\mathbf{j}}_{\mathbf{e}}$,

$$
\left|J_{t} M_{J_{t}}\right\rangle=\sum_{M_{J}, m_{j_{e}}}\left\langle J M_{J} j_{e} m_{j_{e}} \mid J_{t} M_{J_{t}}\right\rangle\left|J M_{J}\right\rangle\left|j_{e} m_{j_{e}}\right\rangle,
$$

in order to rewrite the final state for a specific photoelectron partial wave:

$$
\begin{aligned}
& \left|\psi_{j_{e}}\right\rangle \\
& \quad=\left\langle J j_{e}\left\|D^{k}\right\| J_{0}\right\rangle \sum_{M_{J}, m_{j_{e}}}\left\langle J M_{J} j_{e} m_{j_{e}} \mid J_{t} M_{J_{t}}\right\rangle\left|J M_{J}\right\rangle\left|j_{e} m_{j_{e}}\right\rangle .
\end{aligned}
$$

Here we have introduced additional labels to denote the reduced dipole matrix element for a specific photoelectron partial wave $j_{e}$ and a specific fine-structure ionic state $J$.

For the possibility of multiple photoelectron partial waves, we sum in order to obtain the overall final state

$|\psi\rangle=\sum_{j_{e}} a_{j_{e}} \sum_{M_{J} m_{j_{e}}}\left\langle J M_{J} j_{e} m_{j_{e}} \mid J_{t} M_{J_{t}}\right\rangle\left|J M_{J}\right\rangle\left|j_{e} m_{j_{e}}\right\rangle$,

where we have written $a_{j_{e}} \equiv\left\langle J, j_{e}\left\|D^{k}\right\| J_{0}\right\rangle$ to denote the reduced dipole matrix elements, or in other words the probability amplitudes, for a specific photoelectron partial wave of angular momentum $j_{e}$. Thus, the orientation and alignment parameters allow us to relate measurements of $P_{3}$ and $P_{4}$ to the magnetic sub-state distribution of the ion and subsequently, through the angular momentum coupling of the final state $|\psi\rangle$, to the photoelectron partial-wave probabilities $\left|a_{j_{e}}\right|^{2}$.

As an example, consider an excited ionic state with $J=\frac{1}{2}$ so that

$$
O_{0}\left(J=\frac{1}{2}\right)=\frac{\sum_{M_{J}=-\frac{1}{2}}^{+\frac{1}{2}} M_{J}\left|\left\langle J M_{J} \mid \psi\right\rangle\right|^{2}}{\frac{1}{2}\left(\frac{1}{2}+1\right)} .
$$

In this case, $\frac{3}{4} O_{0}=\frac{1}{2}\left|\left\langle J=\frac{1}{2}, M_{J}=+\frac{1}{2} \mid \psi\right\rangle\right|^{2}-$ $\frac{1}{2}\left|\left\langle J=\frac{1}{2}, M_{J}=-\frac{1}{2} \mid \psi\right\rangle\right|^{2}$. For an ionic state with $J=\frac{1}{2}$, the dipole operator restricts $j_{e}$ to be $1 / 2$ or $3 / 2$ so that the final state can be explicitly written as

$$
\begin{aligned}
|\psi\rangle & =\sum_{j_{e}=\frac{1}{2}}^{\frac{3}{2}} a_{j_{e}} \sum_{M_{J}=-\frac{1}{2}}^{+\frac{1}{2}} \sum_{m_{j_{e}}}\left\langle J=\frac{1}{2}, M_{J} j_{e} m_{j_{e}} \mid J_{t}=1, M_{J_{t}}=1\right\rangle \\
& \times\left|J=\frac{1}{2}, M_{J}\right\rangle\left|j_{e} m_{j_{e}}\right\rangle \\
= & a_{\frac{1}{2}} \sum_{M_{J}=-\frac{1}{2}}^{+\frac{1}{2}} \sum_{m_{j_{e}}=-\frac{1}{2}}^{+\frac{1}{2}}\left\langle\frac{1}{2}, M_{J}, j_{e}=\frac{1}{2}, m_{j_{e}} \mid 1,1\right\rangle \\
& \times\left|\frac{1}{2}, M_{J}\right\rangle\left|j_{e}=\frac{1}{2}, m_{j_{e}}\right\rangle \\
& +a_{\frac{3}{2}} \sum_{M_{J}=-\frac{1}{2}}^{+\frac{1}{2}} \sum_{m_{e}=-\frac{3}{2}}^{+\frac{3}{2}}\left\langle\frac{1}{2}, M_{J}, j_{e}=\frac{3}{2}, m_{j_{e}} \mid 1,1\right\rangle \\
& \times\left|\frac{1}{2}, M_{J}\right\rangle\left|j_{e}=\frac{3}{2}, m_{j_{e}}\right\rangle \\
= & a_{\frac{1}{2}} \sum_{m_{j_{e}}=-\frac{1}{2}}^{+\frac{1}{2}}\left\langle\frac{1}{2}, M_{J}=-\frac{1}{2}, \frac{1}{2}, m_{j_{e}} \mid 1,1\right\rangle \\
& \times\left|\frac{1}{2}, M_{J}=-\frac{1}{2}\right\rangle\left|\frac{1}{2}, m_{j_{e}}\right\rangle \\
& \times\left|\frac{1}{2}, M_{\frac{1}{2}} \sum_{m_{j_{e}}=-\frac{1}{2}}^{+\frac{1}{2}}\left\langle\frac{1}{2}, M_{J}=\frac{1}{2}\right\rangle \frac{1}{2}, m_{j_{e}}\right\rangle
\end{aligned}
$$




$$
\begin{aligned}
& +a_{\frac{3}{2}} \sum_{m_{j_{e}}=-\frac{3}{2}}^{+\frac{3}{2}}\left\langle\frac{1}{2}, M_{J}=-\frac{1}{2}, \frac{3}{2}, m_{j_{e}} \mid 1,1\right\rangle \\
& \times\left|\frac{1}{2}, M_{J}=-\frac{1}{2}\right\rangle\left|\frac{3}{2}, m_{j_{e}}\right\rangle \\
& +a_{\frac{3}{2}} \sum_{m_{j_{e}}=-\frac{3}{2}}^{+\frac{3}{2}}\left\langle\frac{1}{2}, M_{J}=\frac{1}{2}, \frac{3}{2}, m_{j_{e}} \mid 1,1\right\rangle\left|\frac{1}{2}, M_{J}=\frac{1}{2}\right\rangle \\
& \times\left|\frac{3}{2}, m_{j_{e}}\right\rangle .
\end{aligned}
$$

Therefore,

$$
\begin{aligned}
& \left\langle\frac{1}{2}, M_{J}=\frac{1}{2} \mid \psi\right\rangle \\
& =a_{\frac{1}{2}} \sum_{m_{j_{e}}=-\frac{1}{2}}^{+\frac{1}{2}}\left\langle\frac{1}{2}, M_{J}=\frac{1}{2}, \frac{1}{2}, m_{j_{e}} \mid 1,1\right\rangle\left|\frac{1}{2}, m_{j_{e}}\right\rangle \\
& \quad+a_{\frac{3}{2}} \sum_{m_{j_{e}}=-\frac{3}{2}}^{+\frac{3}{2}}\left\langle\frac{1}{2}, M_{J}=\frac{1}{2}, \frac{3}{2}, m_{j_{e}} \mid 1,1\right\rangle\left|\frac{3}{2}, m_{j_{e}}\right\rangle
\end{aligned}
$$

and

$$
\begin{aligned}
& \left\langle\frac{1}{2}, M_{J}=-\frac{1}{2} \mid \psi\right\rangle \\
& =a_{\frac{1}{2}} \sum_{m_{j_{e}}=-\frac{1}{2}}^{+\frac{1}{2}}\left\langle\frac{1}{2}, M_{J}=-\frac{1}{2}, \frac{1}{2}, m_{j_{e}} \mid 1,1\right\rangle\left|\frac{1}{2}, m_{j_{e}}\right\rangle \\
& \quad+a_{\frac{3}{2}} \sum_{m_{j_{e}}=-\frac{3}{2}}^{+\frac{3}{2}}\left\langle\frac{1}{2}, M_{J}=-\frac{1}{2}, \frac{3}{2}, m_{j_{e}} \mid 1,1\right\rangle\left|\frac{3}{2}, m_{j_{e}}\right\rangle .
\end{aligned}
$$

Upon forming the absolute squares we obtain

$$
\begin{aligned}
& \left|\left\langle\frac{1}{2}, M_{J}=\frac{1}{2} \mid \psi\right\rangle\right|^{2}=\left|a_{\frac{1}{2}}\right|^{2} \underbrace{\left\langle\frac{1}{2}, \frac{1}{2}, \frac{1}{2}, m_{j_{e}}=-\frac{1}{2}\right| 1,1}_{0}\rangle^{2} \\
& +\left|a_{\frac{1}{2}}\right|^{2} \underbrace{\left\langle\frac{1}{2}, \frac{1}{2}, \frac{1}{2}, m_{j_{e}}=+\frac{1}{2} \mid 1,1\right\rangle^{2}}_{1} \\
& +\left|a_{\frac{3}{2}}\right|^{2} \underbrace{\left\langle\frac{1}{2}, \frac{1}{2}, \frac{3}{2}, m_{j_{e}}=-\frac{3}{2} \mid 1,1\right\rangle^{2}}_{0} \\
& +\left|a_{\frac{3}{2}}\right|^{2} \underbrace{\left\langle\frac{1}{2}, \frac{1}{2}, \frac{3}{2}, m_{j_{e}}=-\frac{1}{2} \mid 1,1\right\rangle^{2}}_{0} \\
& +\left|a_{\frac{3}{2}}\right|^{2} \underbrace{\langle\underbrace{}_{\frac{1}{2}}, \frac{1}{2}, \frac{3}{2}, m_{j_{e}}=+\frac{1}{2} \mid 1,1\rangle^{2}}_{\frac{1}{2}} \\
& +\left|a_{\frac{3}{2}}\right|^{2} \underbrace{\left\langle\frac{1}{2}, \frac{1}{2}, \frac{3}{2}, m_{j_{e}}=+\frac{3}{2} \mid 1,1\right\rangle^{2}}_{0} \\
& =\left|a_{\frac{1}{2}}\right|^{2}+\frac{1}{4}\left|a_{\frac{3}{2}}\right|^{2}
\end{aligned}
$$

and

$\left|\left\langle\frac{1}{2}, M_{J}=-\frac{1}{2} \mid \psi\right\rangle\right|^{2}=\left|a_{\frac{1}{2}}\right|^{2} \underbrace{\left\langle\frac{1}{2},-\frac{1}{2}, \frac{1}{2}, m_{j_{e}}=-\frac{1}{2} \mid 1,1\right\rangle^{2}}_{0}$

$$
\begin{aligned}
& +\left|a_{\frac{1}{2}}\right|^{2} \underbrace{\left\langle\frac{1}{2},-\frac{1}{2}, \frac{1}{2}, m_{j_{e}}=+\frac{1}{2} \mid 1,1\right\rangle^{2}}_{0} \\
& +\left|a_{\frac{3}{2}}\right|^{2} \underbrace{\left\langle\frac{1}{2},-\frac{1}{2}, \frac{3}{2}, m_{j_{e}}=-\frac{3}{2} \mid 1,1\right\rangle^{2}}_{0} \\
& +\left|a_{\frac{3}{2}}\right|^{2} \underbrace{\left\langle\frac{1}{2},-\frac{1}{2}, \frac{3}{2}, m_{j_{e}}=-\frac{1}{2} \mid 1,1\right\rangle^{2}}_{0} \\
& +\left|a_{\frac{3}{2}}\right|^{2} \underbrace{\left\langle\frac{1}{2},-\frac{1}{2}, \frac{3}{2}, m_{j_{e}}=+\frac{1}{2} \mid 1,1\right\rangle^{2}}_{0} \\
& +\left|a_{\frac{3}{2}}\right|^{2} \underbrace{\left\langle\frac{1}{2},-\frac{1}{2}, \frac{3}{2}, m_{j_{e}}=+\frac{3}{2} \mid 1,1\right\rangle^{2}}_{-\frac{\sqrt{3}}{2}} \\
& =\frac{3}{4}\left|a_{\frac{3}{2}}\right|^{2} \text {. }
\end{aligned}
$$

Therefore

$$
\begin{aligned}
\frac{3}{4} O_{0}= & \frac{1}{2}\left|\left\langle J=\frac{1}{2}, M_{J}=+\frac{1}{2} \mid \psi\right\rangle\right|^{2} \\
& -\frac{1}{2}|| J=\frac{1}{2}, M_{J}=-\left.\frac{1}{2}|\psi\rangle\right|^{2} \\
= & \frac{1}{2}\left(\left|a_{\frac{1}{2}}\right|^{2}+\frac{1}{4}\left|a_{\frac{3}{2}}\right|^{2}\right)-\frac{1}{2}\left(\frac{3}{4}\left|a_{\frac{3}{2}}\right|^{2}\right) \\
= & \frac{1}{2}\left|a_{\frac{1}{2}}\right|^{2}-\frac{1}{4}\left|a_{\frac{3}{2}}\right|^{2} .
\end{aligned}
$$
yields

Imposing the normalization condition $\left|a_{\frac{1}{2}}\right|^{2}+\left|a_{\frac{3}{2}}\right|^{2}=1$

$$
\left|a_{\frac{1}{2}}\right|^{2}=\frac{1}{3}+O_{0}\left(J=\frac{1}{2}\right)
$$

and

$$
\left|a_{\frac{3}{2}}\right|^{2}=\frac{2}{3}-O_{0}\left(J=\frac{1}{2}\right) .
$$

This example involves only the orientation parameter since the alignment parameter is identically zero for a state with $J=\frac{1}{2}$. From equations (21), (22), (24) and (25) one can obtain the fractional probabilities of individual $M_{\mathrm{J}}$ states within the excited ion. These probabilities are proportional to $O_{o}(J)$ plus a constant.

For the case of an ionic state with $J>\frac{1}{2}$ there are three photoelectron partial waves possible with different total angular momenta $j_{e}$; the additional linear polarization measurement along with the corresponding alignment parameter for this case provides the additional equation to extract all three partial-wave probabilities. The most general form of these equations was given in [18]. After revising those equations to account for the different (Fano-Macek) convention used in the present study, and also after correcting the third of equation (54) of [18] for a typographical error in the sign shown for the orientation term, the general relationship is given by

$$
\begin{gathered}
\sigma\left(J ; j_{e}=J+1\right)=\frac{5 J}{3(2 J+1)} A_{0}(J) \\
-\frac{(2 J+3) J}{2 J+1} O_{0}(J)+\frac{2 J+3}{3(2 J+1)}, \\
\sigma\left(J ; j_{e}=J-1\right)=\frac{5(J+1)}{3(2 J+1)} A_{0}(J) \\
+\frac{(2 J-1)(J+1)}{2 J+1} O_{0}(J)+\frac{2 J-1}{3(2 J+1)}
\end{gathered}
$$


and

$$
\sigma\left(J ; j_{e}=J\right)=-\frac{5}{3} A_{0}(J)+O_{0}(J)+\frac{1}{3} .
$$

Note that for an initial target state with zero angular momentum $J_{0}=0$, as is the case here, the angular momentum transfer $j_{\mathrm{t}}$ of [18] reduces simply to the photoelectron total angular momentum $j_{\mathrm{e}}$.

\subsection{Theoretical calculations}

Photoionization cross sections for Ar have also been obtained theoretically using the ab-initio $R$-matrix approach [19], which was extended to use multi-configuration Hartree-Fock (MCHF) orbitals for the description of residual states of Ar II [20]. A detailed description of how photoionization cross sections are determined in $L S$-coupling has been given previously [21, 22]. However, as mentioned above, none of the final Ar II states studied here, apart from the ${ }^{4} \mathrm{P}_{5 / 2}^{\circ}$ level, can be described well in $L S$-coupling. We have therefore incorporated the spin-orbit splitting of the individual Ar II states in the present calculations through an $L S$-to-jj frame transformation $[19,23,24]$. In addition, spin-orbit interactions between different states in the $3 \mathrm{p}^{4}\left({ }^{3} \mathrm{P}\right) 4 \mathrm{p}$ configuration cannot be neglected. We have thus added a second frame transformation to take spin-orbit interactions between Ar II levels into account $[23,24]$.

We start the calculations by determining physical $1 \mathrm{~s}, 2 \mathrm{~s}$, $2 p, 3 s, 3 p, 3 d, 4 s$ and $4 p$ orbitals for Ar II using the MCHF approach [25]. To improve the description of the Ar II states, we also obtain unphysical $4 \mathrm{~d}, 5 \mathrm{~s}$ and $5 \mathrm{p}$ correlation orbitals. Using these orbitals, we generate an extensive basis for the description of the $3 s^{2} 3 p^{5}, 3 s 3 p^{6}$, and all $3 s^{2} 3 p^{4}(3 d, 4 s, 4 p)$ states of Ar II. The photoionization cross sections are obtained using $R$-matrix theory. Within the inner region, a sphere with a radius of $13 \mathrm{au}$, we generate a basis set for Ar by combining all states within the Ar II basis with a complete set of single-electron continuum functions. The maximum angular momentum for the continuum electrons is three. The initial state of Ar is given by the eigenfunction associated with the lowest eigenvalue with ${ }^{1} \mathrm{~S}^{\mathrm{e}}$ symmetry. To improve the agreement with experiment we need the ionization thresholds in theory and experiment to coincide, and the energies of this Ar ground state and all Ar II thresholds are thus shifted to their experimental values.

For the final-state calculations, we include not only the ${ }^{1} \mathrm{P}^{\circ}$ symmetry, but also the ${ }^{3} \mathrm{~S}^{\circ},{ }^{3} \mathrm{P}^{\circ},{ }^{3} \mathrm{D}^{\circ},{ }^{5} \mathrm{P}^{\circ},{ }^{5} \mathrm{D}^{\circ}$ and ${ }^{5} \mathrm{~F}^{\circ}$ symmetries which are coupled to the ${ }^{1} \mathrm{P}^{\circ}$ symmetry (either directly or indirectly) by spin-orbit interactions. For each of these symmetries, we obtain $K$-matrices and dipole vectors at a range of energies by matching the inner region solutions to asymptotic solutions in the outer region. Application of the $L S$-jj frame transformation gives dipole vectors and $K$ matrices which include the spin-orbit interactions within the Ar II states. Finally, the frame transformation coupling the various levels of $\mathrm{Ar}$ II then gives dipole vectors and $K$-matrices including spin-orbit interactions between the different Ar II levels. From these, we can then determine the photoionizationexcitation cross section for each Ar II level.

To compare the theoretical photoionization cross sections with those obtained experimentally, we convolve the theoretical cross sections with a Gaussian having a fullwidth at half-maximum between 5 and $10 \mathrm{meV}$, corresponding to the respective experimental resolutions of $10 \mathrm{meV}$ for reaction (1), $8 \mathrm{meV}$ for reactions (2)-(4), and $5 \mathrm{meV}$ for reactions (5) and (6). This is of particular importance for comparison with experiment at the present photon energies. The final states contain many overlapping Rydberg series, and the resolution determines which Rydberg series will be observed at a particular photon energy.

\section{Discussion of results}

\subsection{Overview}

Figure 3 (whose data were originally published in [12]) shows the total relative intensity (in arbitrary units) of the $476.5 \mathrm{~nm}$ fluorescence from the $\mathrm{Ar}^{+} 3 \mathrm{p}^{4}\left[{ }^{3} \mathrm{P}\right] 4 \mathrm{p}^{2} \mathrm{P}_{3 / 2}^{\circ}-4 \mathrm{~s}\left[{ }^{3} \mathrm{P}\right]{ }^{2} \mathrm{P}_{1 / 2}$ transition (reaction (2)). The lines connecting the experimental data points of figure 3 are drawn to guide the eye. This intensity, $I=I_{\|}+2 I_{\perp}$, which combines the linearly polarized fluorescence intensities parallel and perpendicular to the incident photon beam, is proportional to the total excitation cross section. Figure 3 sets the context for discussion of the rest of our data and bears some discussion. The threshold for this simultaneous excitation/ionization reaction populating the $3 \mathrm{p}^{4}$ $\left[{ }^{3} \mathrm{P}\right] 4 \mathrm{p}^{2} \mathrm{P}_{3 / 2}^{\circ}$ state is evident at $35.625 \mathrm{eV}$, which is consistent, given the $8 \mathrm{meV}$ resolution of the data, with the spectroscopic value of $35.627 \mathrm{eV}$ obtained by Minnhagen [13]. (We estimate an absolute energy uncertainty in all the data presented here of $\pm 1 \mathrm{meV}$. The estimated doubly excited resonance positions have higher uncertainty, as discussed below.) Overlain in figure 3 are our estimated positions of the terms corresponding to five doubly excited Ar I Rydberg series. These were obtained from the high-resolution absorption data of Madden, Ederer and Codling [1], using known series limits and accepted ranges for quantum defects [12] in the formula [26]

$$
E(n)=\frac{R y_{\infty}}{(n-\delta(l, n))^{2}},
$$

where $\delta(l, n)=\left(k_{1}(l)+k_{2}(l) / n^{2}\right)$ and $k_{1}$ and $k_{2}$ are weak functions of $l$. These estimated resonance positions correlate well with the observed structure in the spectra. Parity and angular momentum conservation restrict the orbital angular momentum of the Rydberg electron to only those values indicated for each of the series limits shown.

The complex structure of this spectrum is not surprising, given the large number of doubly-excited states that can be accessed in this energy range that autoionize into the final ionic state. The dynamical processes leading to the production of the Ar II $3 \mathrm{p}^{4}\left[{ }^{3} \mathrm{P}\right] 4 \mathrm{p}^{2} \mathrm{P}_{3 / 2}^{\circ}$ state are complicated by the fact that the photoelectron leaves, over this energy range, with at most $\sim 1 \mathrm{eV}$, meaning that post-collision interactions will be very important.

Despite its complicated structure, cross sections for photoionization of Ar with the excitation of the $3 p^{4}\left({ }^{3} \mathrm{P}\right) 4 \mathrm{p}$ ${ }^{2} \mathrm{P}^{\circ}$ state were obtained with encouraging accuracy by van der Hart and Greene [22], albeit with some discrepancies in, e.g., the region near $35.76 \mathrm{eV}$. These cross sections were obtained within an $L S$-coupling approximation, and under 


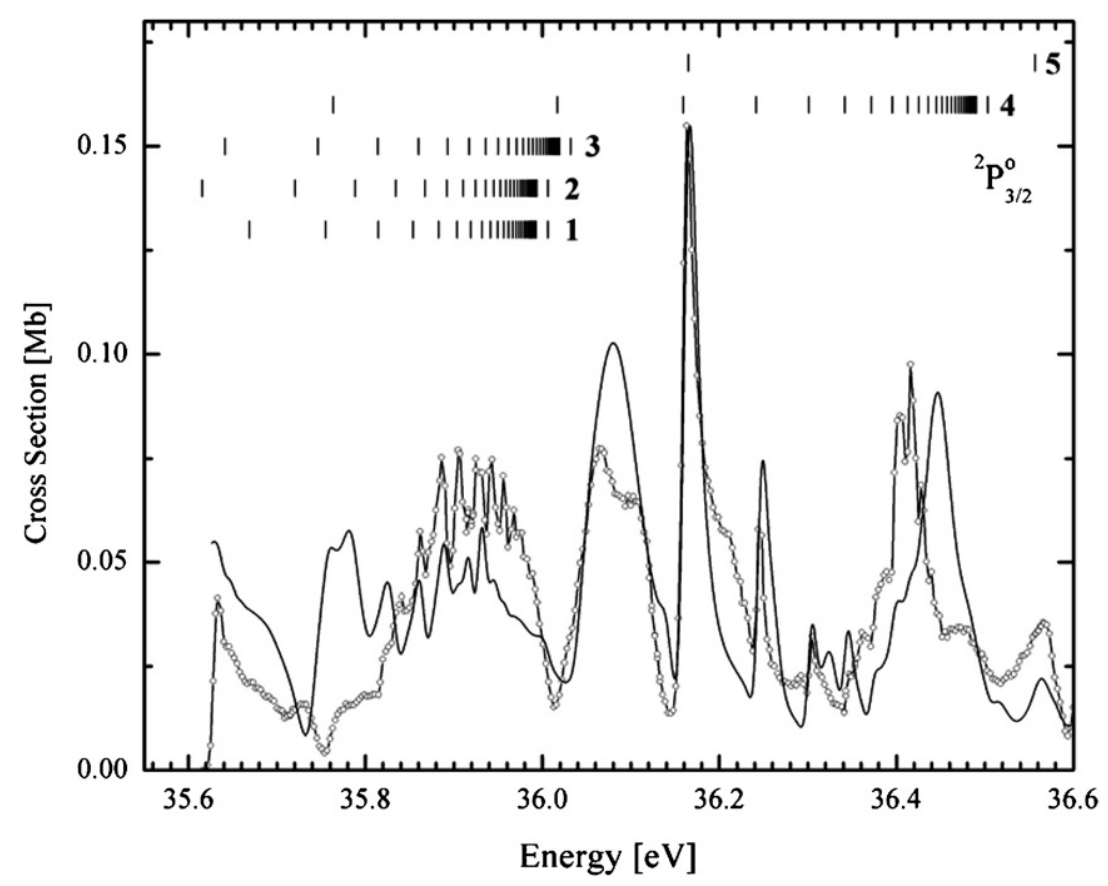

Figure 3. Total fluorescent intensity for reaction (2) (476.5 nm; Ar II $3 \mathrm{p}^{4}\left[{ }^{3} \mathrm{P}\right] 4 \mathrm{p}^{2} \mathrm{P}_{3 / 2}^{\circ}-\left[{ }^{3} \mathrm{P}\right] 4 \mathrm{~s}^{2} \mathrm{P}_{1 / 2}$ ). The experimental data (open circles) are normalized to theory (solid line) of this work at the energy of the peak of the $\operatorname{Ar} I\left(3 s^{2} 3 p^{4}\right)\left[{ }^{1} S\right] 4 s^{2} S$ resonance at $36.163 \mathrm{eV}$. The vertical lines show the positions of resonant doubly excited autoionizing states of Ar I: (1) $3 \mathrm{p}^{4}\left[{ }^{1} \mathrm{D}\right] 3 \mathrm{~d}^{2} \mathrm{~F}_{5 / 2} n \mathrm{p}$; (2) $3 \mathrm{p}^{4}\left[{ }^{1} \mathrm{D}\right] 3 \mathrm{~d}^{2} \mathrm{~F}_{5 / 2} n \mathrm{f}$; (3) $3 \mathrm{p}^{4}\left[{ }^{1} \mathrm{D}\right] 3 \mathrm{~d}^{2} \mathrm{~F}_{7 / 2} n \mathrm{f}$; (4) $3 \mathrm{p}^{4}\left[{ }^{1} \mathrm{~S}\right] 4 \mathrm{~s}^{2} \mathrm{~S}_{1 / 2} n \mathrm{p}$; and (5) $3 \mathrm{p}^{4}\left[{ }^{1} \mathrm{D}\right] 4 \mathrm{p}^{2} \mathrm{P}_{1 / 2} n \mathrm{~s}$ (see the text). The experimental resolution and convolution width of theory are $8 \mathrm{meV}$.

the assumption of a $60 \mathrm{meV}$ photon-energy resolution. The present experiments have significantly better resolution, and in figure 3, we thus present our new theoretical cross sections convoluted with a $8 \mathrm{meV}$ photon-energy resolution. They have been obtained following the $L S$-to-jj frame transformation discussed above, and thus include spin-orbit mixing of the different Ar II levels. Considering the fact that the present spectrum contains many overlapping Rydberg series, the theoretical cross sections are in quite reasonable agreement with experiment.

The data shown in figure 3 are dominated by (1) an obscured Rydberg series of resonances between 35.85 and $36.0 \mathrm{eV}$, most likely a combination of resonances from the three $\left[{ }^{1} \mathrm{D}\right] 3 \mathrm{~d}^{2} \mathrm{~F}$ series shown in figure $3,(2)$ a number of fairly well-isolated, prominent features between 36.0 and $36.35 \mathrm{eV}$, and (3) a second apparent partial Rydberg series between 36.34 and $36.46 \mathrm{eV}$, most likely belonging to the $\left[{ }^{1} \mathrm{~S}\right] 4 \mathrm{~s}^{2} \mathrm{~S}$ Ar II series limit, with a significant background just below and above $36.4 \mathrm{eV}$. Both Rydberg series ((1) and (3) above) appear to be made up of symmetric peaks, to the extent that they are resolved. The nature of the background underlying these series is not readily identifiable. In [22], the lower-energy background between $35.7 \mathrm{eV}$ and $36 \mathrm{eV}$ is attributed to 'the Rydberg series leading up to the ${ }^{2} \mathrm{~S}^{\mathrm{e}}$ threshold' (series label ' 4 ' in figure 3). Another possibility is that the background is simply the unresolved structure of the doubly excited Rydberg series labelled ' 1 ', ' 2 ' ' and ' 3 ' in figure 3 with the $7 \mathrm{p}\left[{ }^{1} \mathrm{~S}\right] 4 \mathrm{~s}$ ${ }^{2} \mathrm{~S}$ (Rydberg series (4)) term at $36.02 \mathrm{eV}$ being observed as a window resonance. We will discuss this issue in more detail below.
The prominent maxima at $36.16,36.25$, and $36.30 \mathrm{eV}$ are clearly the $8 \mathrm{p}, 9 \mathrm{p}$ and $10 \mathrm{p}$ members of the $\left[{ }^{1} \mathrm{~S}\right] 4 \mathrm{~s}^{2} \mathrm{~S}$ $n^{\prime} \mathrm{p}$ series. It is interesting to note that of all the $3 \mathrm{~s}^{2} 3 \mathrm{p}^{4} n l$, $n^{\prime} l^{\prime} \mathrm{Ar}^{* *}$ Rydberg series identified by Madden, Ederer and Codling [1] and by Baig et al [2], this is the only one with $n l=4 \mathrm{~s}$. Most importantly, the $8 \mathrm{p}, 9 \mathrm{p}$ and $10 \mathrm{p}$ resonances display a clear asymmetry reminiscent of a Beutler-Fano profile, indicating interference with other ionization pathways with nearly constant amplitude over the energy width of the resonance. It is the nature of this interference that we are most concerned with in the current work. Finally, the double-humped peaks at 36.07 and $36.11 \mathrm{eV}$, seen as a single maximum in the theoretical spectrum, are not readily identifiable in terms of the Rydberg series shown in figure 3, but will also be discussed further below.

\subsection{The region between 36.00 and $36.35 \mathrm{eV}$}

Because of the prominence, unique configuration and asymmetric character of the resonances in the region between 36.0 and $36.35 \mathrm{eV}$, we have investigated this range in more detail, measuring the relative intensities of the reaction pathways (1)-(6) listed in the introduction over this energy range. These residual ionic states were chosen because the first four match the $3 s^{2} 3 p^{4}\left[{ }^{3} \mathrm{P}\right] 4 \mathrm{p}{ }^{2} \mathrm{P},{ }^{2} \mathrm{D} L S$ channels investigated by van der Hart and Greene [22], and because the last two reactions producing quartet states hold the promise of providing additional insight into spin-orbit forces during the photoionization/excitation process. The relative fluorescence intensity for all six excited states of Ar II are shown in figure 4, in conjunction with the theoretical results. Experimental 
results for both the ${ }^{2} \mathrm{P}$ states have been published previously [9-12]. Theoretical cross sections for the ${ }^{4} \mathrm{P}_{5 / 2}^{\circ}$ and ${ }^{2} \mathrm{P}_{3 / 2}^{\circ}$ states have also been published previously [24]. The theoretical cross sections include the spin-orbit couplings between Ar II states. For most of the present spectra, this coupling has relatively little effect, affecting resonance heights by up to about $30 \%$. However, there are some more noticeable effects. In the ${ }^{2} \mathrm{P}^{\circ}{ }_{1 / 2}$ spectrum, the peak at $36.05 \mathrm{eV}$ decreases by a factor of two when the coupling is taken into account.

Spin-orbit coupling between Ar II states has, however, a dramatic effect on the photoionization-excitation spectrum for the ${ }^{4} \mathrm{D}^{\circ}{ }_{5 / 2}$ symmetry. In the absence of spin-orbit mixing between Ar II states, the maximum for the photoionization cross section in the photon-energy range between $36 \mathrm{eV}$ and $36.36 \mathrm{eV}$ equals $6 \mathrm{kB}$ at a photon energy of $36.08 \mathrm{eV}$. No evidence of a Rydberg series converging to the ${ }^{2} S^{e}$ threshold is seen. Figure 4 shows the effect on the theoretical spectrum when spin-orbit coupling between Ar II states is taken into account. The Rydberg series leading up to the ${ }^{2} S^{e}$ threshold now appears prominently in the theoretical spectrum, in line with the experimental observations. The main reason for this is that the ${ }^{4} \mathrm{D}^{\circ}{ }_{5 / 2}$ state of $\mathrm{Ar}$ II has approximately a $16 \%$ contribution from the ${ }^{2} \mathrm{D}^{\circ}{ }_{5 / 2}$ state.

As mentioned above, and with the exception of the ${ }^{4} \mathrm{P}^{\circ}{ }_{5 / 2}$ data (reaction (5)), the $\left[{ }^{1} \mathrm{~S}\right] 4 \mathrm{~s}^{2} \mathrm{~S} n \mathrm{p}$ Rydberg series dominates these spectra. This dominance is somewhat counterintuitive, since this series autoionizes to the observed Ar II states, a process that must involve the $3 p$ electrons, since the $3 p^{4}$ core changes from ${ }^{1} \mathrm{~S}$ to ${ }^{3} \mathrm{P}$ coupling. Therefore, the autoionization process proceeds predominantly via an interaction whereby the $4 \mathrm{~s}$ electron is ejected in combination with a symmetry change of the core. The outer electron is a spectator experiencing a sudden change in the potential. The transition amplitude thus includes an overlap between the $n p$ orbital before and the $4 p$ orbital after autoionization. A second gross feature of the data is the qualitative similarity between the ${ }^{2} \mathrm{P}$ and ${ }^{2} \mathrm{D}$ spectra. This applies both to the theoretical and experimental spectra. Within the former, the most important difference is the appearance of a resonance at $36.27 \mathrm{eV}$ in the ${ }^{2} \mathrm{P}^{\circ}{ }_{1 / 2}$ spectrum, which is absent in the ${ }^{2} \mathrm{P}_{3 / 2}^{\circ}$ spectrum. This suggests that spinorbit interactions wield some influence on the photoionization process for allowed channels in $L S$-coupling.

The theoretical data can be used to estimate the importance of spin-orbit interactions within individual Ar II states on the photoionization spectra, and the importance of spin-orbit coupling between the Ar II states. The ${ }^{4} \mathrm{P}^{\circ} 5 / 2$ state has a contribution of about $3 \%$ from ${ }^{4} \mathrm{D}^{\circ}{ }_{5 / 2}$ and $0.15 \%$ from ${ }^{2} \mathrm{D}^{\circ}{ }_{5 / 2}$ [14]. This latter contribution could lead to the $\left[{ }^{1} \mathrm{~S}\right] 4 \mathrm{~s}^{2} \mathrm{~S} n \mathrm{p}$ Rydberg series, but its small magnitude means that it cannot be observed. On the other hand, the ${ }^{4} \mathrm{D}^{\circ}{ }_{5 / 2}$ state has a contribution of about $15 \%$ from ${ }^{2} \mathrm{D}^{\circ}{ }_{5 / 2}$ and $1 \%$ from ${ }^{4} \mathrm{P}^{\circ}{ }_{5 / 2}$. The large contribution from the ${ }^{2} \mathrm{D}^{\circ}{ }_{5 / 2}$ state leads to the pronounced $\left[{ }^{1} \mathrm{~S}\right] 4 \mathrm{~s}^{2} \mathrm{~S} n \mathrm{p}$ Rydberg series. The agreement between theory and experiment thus gives a rough gauge of the importance of spin-orbit interactions on the photoionization processes, as well as information on the nature of the important spin-orbit interactions.

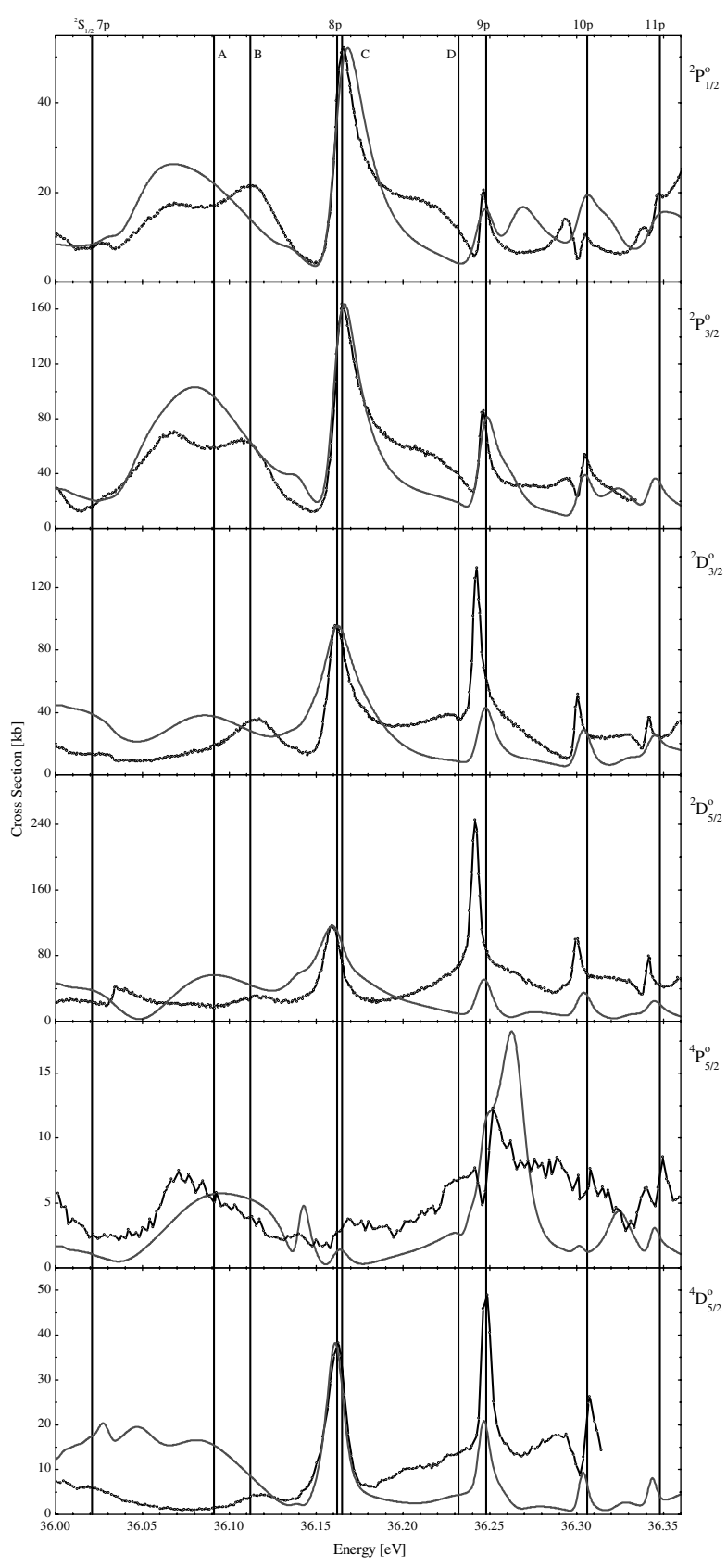

Figure 4. Relative total fluorescent intensity for reactions (1)-(6) (open circles) normalized to the absolute theoretical calculations of this work (thin smooth lines) at the energy of the peak of the Ar I $\left(3 s^{2} 3 p^{4}\right)\left[{ }^{1} S\right] 4 s^{2} S$ p resonance maximum at $36.163 \mathrm{eV}$, except for reaction (5) which is normalized at the peak of the experimental data. The lines labelled '7'-'11' indicate the Rydberg series Ar I $\left(3 \mathrm{~s}^{2} 3 \mathrm{p}^{4}\right)\left[{ }^{1} \mathrm{~S}\right] 4 \mathrm{~s}^{2} \mathrm{~S} n \mathrm{p}$ with $n=7,8,9,10$, and 11 at $36.021,36.162$, $36.249,36.306$ and $36.348 \mathrm{eV}$, respectively. These energies are those measured in the absorption data of [2], with an uncertainty of about $1 \mathrm{meV}$. The four labelled resonances correspond to states whose energies are inferred from the positions of other members of the same series in absorption measurements $[1,2]$, with a corresponding uncertainty in energy of $\sim 25 \mathrm{meV}$ due to the extrapolated fit and uncertainty in the quantum defects corresponding to these series (equation (29)). These states are: (A), $\left(3 \mathrm{~s}^{2} 3 \mathrm{p}^{4}\right)\left[{ }^{1} \mathrm{D}\right] 4 \mathrm{p}^{2} \mathrm{P}_{3 / 2} 6 \mathrm{~s}$ (36.091 eV); (B), $\left(3 \mathrm{~s}^{2} 3 \mathrm{p}^{4}\right)\left[{ }^{1} \mathrm{D}\right] 3 \mathrm{~d}^{2} \mathrm{P}_{3 / 2} 5 \mathrm{p}$ (36.112 eV); (C), $\left(3 \mathrm{~s}^{2} 3 \mathrm{p}^{4}\right)\left[{ }^{1} \mathrm{D}\right] 4 \mathrm{p}^{2} \mathrm{P}_{1 / 2} 6 \mathrm{~s}(36.165 \mathrm{eV})$; and (D), $\left(3 \mathrm{~s}^{2} 3 \mathrm{p}^{4}\right)\left[{ }^{1} \mathrm{D}\right] 4 \mathrm{p}$ ${ }^{2} \mathrm{D}_{3 / 2} 6 \mathrm{~s}(36.232 \mathrm{eV})$. The experimental resolution and convolution width of theory are $10 \mathrm{meV}$ for reaction (1), $8 \mathrm{meV}$ for reactions (2)-(4), and $5 \mathrm{meV}$ for reactions (5) and (6), respectively. 


\subsection{Interference effects}

We now consider these data in the context of interference phenomena involving both 'direct' ionization (in which no double excitation of the neutral target occurs), and resonance phenomena. We will refer to an 'outgoing channel' as a specific fine-structure state of the residual ion in conjunction with an outgoing electron having partial-wave quantum numbers $l_{\mathrm{j}}$. The intensity data we have considered so far is an incoherent sum over the various partial waves. On the other hand, a given outgoing channel amplitude is a coherent sum of both direct and resonant processes. There is only one direct process, but any doubly excited states that autoionize to the final channel and that can be excited within their natural widths may also contribute coherently to the light we see from the residual ion. A three-path interference of this type can be written as

$$
\gamma+\operatorname{Ar} \rightarrow\left\{\begin{array}{c}
\operatorname{Ar}^{* *}(1) \\
\operatorname{Ar}^{* *}(2) \\
\operatorname{Ar}^{*+}(3)+\mathrm{e}^{-}
\end{array}\right\} \rightarrow \operatorname{Ar}^{*+}(3)+\mathrm{e}^{-}
$$

where the numbers in parentheses refer to specific states of $\mathrm{Ar}^{* *}$ or $\mathrm{Ar}^{*+}$.

The question now arises, how important in these data is the direct process, and to what extent do the various amplitudes of the different paths interfere with each other? We note that a clear distinction with respect to interaction times can be drawn between the prominent resonances in our data and any direct photoionization process. Resonances with a width of $\sim 10 \mathrm{meV}$ decay in about $0.1 \mathrm{ps}$, whereas an electron directly liberated by a photon with an energy $0.6 \mathrm{eV}$ above the threshold for the production of a given residual ionic state takes $\sim 1$ fs to escape the atom. A review of all data published to date for Ar II satellite fluorescence [7-12] reveals that with the exception of the ${ }^{2} \mathrm{~S} n \mathrm{p}$ resonances in the energy region considered in figure 4 , there are no manifestly asymmetric peaks. We assume that an asymmetric peak reminiscent of a Beutler-Fano profile is indicative of some kind of interference, whether it be between two resonant states (one of which may be quite broad with a phase that varies slowly with energy), between a resonant state and a direct process, or some combination of the two. A series of reasonably symmetric peaks or features may either represent obscured evidence of interference or simply a series of unresolved non-interfering (because of their separation in energy) resonances. We restrict this discussion to satellite fluorescence; Ar absorption spectra generally indicate asymmetric 'Beutler-Fano'-like profiles, but represent such a large number of direct background and resonance channels that a simple interpretation of any features of this type is virtually impossible.

Theoretical estimates of the direct production cross sections for the $3 p^{4}\left({ }^{3} \mathrm{P}\right) 4 \mathrm{p}$ states imply that they should be much less important in this energy range than resonant processes $[22,27,28]$. Indeed, there is no readily identifiable 'smooth' underlying background in any of these spectra, with the possible exception of the reactions (1) and (2) ${ }^{2} \mathrm{P}$ spectra above $36.26 \mathrm{eV}$, and the reaction $(6){ }^{4} \mathrm{D}_{5 / 2}$ spectrum above $36.07 \mathrm{eV}$. In principle, one could argue that both ${ }^{2} \mathrm{D}$ spectra also have a small direct background component over the entire energy range of figure 4. Having said this, a zeroth-order description of the photoionization process resulting in excited satellite states in this energy regime is that it occurs almost exclusively by resonant double excitation of the ground-state target. (This is in contrast with, e.g., the Ar photoelectron spectra of Canton-Rogan et al [5] taken below the $3 \mathrm{~s}^{-1} 3 \mathrm{p}^{4}$ threshold at $29.2 \mathrm{eV}$, which is dominated by the direct process.) Thus the only clear evidence for interference effects in these spectra is that of the ${ }^{2} \mathrm{~S} n \mathrm{p}$ series interfering with other broad resonances.

The classification of other resonances in this energy range is difficult. While the ${ }^{2} \mathrm{~S} 8 \mathrm{p}, 9 \mathrm{p}, 10 \mathrm{p}$ and $11 \mathrm{p}$ resonances are sharp and prominent, with what resonances are they interfering to produce the asymmetric profiles we observe? In addition, why is the ${ }^{2} \mathrm{~S} 7 \mathrm{p}$ resonance not in evidence (see below)? Using the assignments provided by Madden, Ederer and Codling [1], and using their estimated quantum defects to extrapolate to lower-n resonances, we infer that the $\left[{ }^{1} \mathrm{D}\right]$ $3 \mathrm{~d}^{2} \mathrm{P}_{3 / 2} 5 \mathrm{p}$ resonance (labelled $\mathrm{B}$ in figure 4) should occur in the vicinity of $36.11 \mathrm{eV}$. Another resonance, observed but unidentified in [1] but assigned by us as the $\left[{ }^{1} \mathrm{D}\right] 4 \mathrm{p}^{2} \mathrm{P}_{3 / 2} 6 \mathrm{~s}$ resonance (labelled A), should exist at about $36.09 \mathrm{eV}$. Other features, unobserved in [1] or [2] but extrapolated from series elements they did observe, are the $\left[{ }^{1} \mathrm{D}\right] 4 \mathrm{p}^{2} \mathrm{P}_{1 / 2}$ 6s (label C) and $\left[{ }^{1} \mathrm{D}\right] 4 \mathrm{p}^{2} \mathrm{D}_{3 / 2}$ 6s (label D) resonances at 36.165 and $36.232 \mathrm{eV}$ respectively. Since the four labelled resonances have outer electrons with relatively low principal quantum number, they can be reasonably expected to be broader than those associated with the ${ }^{2} \mathrm{~S}(n>7)$ p series. We find no other experimental evidence in the literature for other resonances in this energy range.

Van der Hart and Greene [22] predicted a broad feature in the vicinity of $36.05 \mathrm{eV}$ in both the ${ }^{2} \mathrm{P}$ and ${ }^{2} \mathrm{D}$ state data, which they assigned as a $\left[{ }^{1} \mathrm{D}\right] 4 \mathrm{p}{ }^{2} \mathrm{P}$ 6s resonance. Using the extrapolation procedure discussed above, this now seems more likely to be a $4 \mathrm{p}^{2} \mathrm{P}$ fine-structure doublet at $36.091 \mathrm{eV}$ $\left({ }^{2} \mathrm{P}_{3 / 2}\right.$; label A) and $36.165 \mathrm{eV}\left({ }^{2} \mathrm{P}_{1 / 2}\right.$; label $\left.\mathrm{C}\right)$. We see a substantially similar feature in the ${ }^{2} \mathrm{P}$ data only, but with a central local minimum at $36.09 \mathrm{eV}$, our proposed position for the A resonance. (Weak corresponding features of these local maxima at 36.065 and $36.11 \mathrm{eV}$ can be seen in all of the other spectra in figure 4). The current theoretical results continue to yield a broad, unbifurcated feature between 36.06 and $36.1 \mathrm{eV}$ in both the ${ }^{2} \mathrm{P}$ and ${ }^{2} \mathrm{D}$ spectra. The predicted relative strengths of this feature in the ${ }^{2} \mathrm{P}$ and ${ }^{2} \mathrm{D}$ spectra agree well with experiment. There would thus appear to be three possibilities in the region between 36.00 and $36.14 \mathrm{eV}$. There may be two fairly broad interfering resonances centered at about $36.09 \mathrm{eV}$, with the $\mathrm{A}$ and $\mathrm{B}$ assignments, one of them acting as a window resonance. Alternately, the A and B features may actually be separated and centered at $\sim 36.07$ and $\sim 36.11 \mathrm{eV}$. Finally, an extremely broad, strong resonance centered at $\sim 36.04 \mathrm{eV}$, with a width of $\sim 0.6 \mathrm{eV}$ may exist. If this is the case the ${ }^{2} \mathrm{~S}$ $7 \mathrm{p}$ resonance is in fact evident as a deep window resonance (seen most clearly in figure 3), and slight perturbations of the broad resonance may be caused by the A and B features. Interference between this big resonance and the higher ${ }^{2} \mathrm{~S}$ $8 p, 9 p$ and possibly $10 p$ resonances could then explain the 
asymmetries of these latter peaks. On the other hand, it is not possible to rule out some interference between the ${ }^{2} \mathrm{~S} n>8$ resonances with a fairly flat direct-process background at energies greater than $\sim 36.2 \mathrm{eV}$, except in the case of the $9 \mathrm{p}$ resonance in the ${ }^{4} \mathrm{P}$ spectrum; this strongly asymmetric profile is probably due to the interference of this resonance with the (broad) D resonance. The ${ }^{2} \mathrm{~S} 9 \mathrm{p}$ resonance is characterized by shapes that are quite similar to Beutler-Fano profiles, except in the ${ }^{2} \mathrm{D}_{3 / 2}$ spectrum, where a small dip at $36.233 \mathrm{eV}$ would appear to be caused by the label-D resonance. Finally, we note that while the ${ }^{2} \mathrm{~S} n=8$ and 9 resonances could be characterized by roughly similar Beutler-Fano ' $\mathrm{q}$ ' shape parameters in the ${ }^{2} \mathrm{P}$ data, this shape changes significantly for $n=10$. (The use of quotation marks about the Beutler-Fano shape parameter will be discussed below.) This is attributed in [22] to interference with a $\left[{ }^{1} \mathrm{D}\right] 3 \mathrm{~d}^{2} \mathrm{D} 4 \mathrm{f}$ resonance. However, no resonance in this series has been observed experimentally.

\subsection{Partial-wave analysis}

We now consider these data in even finer detail by using the procedure outlined above to extract the fractional contributions of each electron partial wave to the total fluorescence intensity. By alternating the orientation of the linear polarizer for the $90^{\circ} P_{4}$ fluorescent detection system of figure 2 , the fluorescent intensity parallel $I_{\|}$and perpendicular $I_{\perp}$ to the $\hat{\mathbf{z}}$ direction was measured at each ionizing photon energy. These individual polarized intensities were normalized to the ionizing photon flux as well as corrected for the extinction ratio of the linear polarizer. In similar fashion, the normalized fluorescent intensity with right- $\left(I_{\mathrm{RHH}}\right)$ and left-handed helicity $\left(I_{\mathrm{LHH}}\right)$ were measured. Repeating the measurement upon incrementing the ionizing photon energy resulted in the

$$
P_{4} \equiv\left(I_{\|}-I_{\perp}\right) /\left(I_{\|}+I_{\perp}\right)
$$

and

$$
P_{3} \equiv\left(I_{\mathrm{RHH}}-I_{\mathrm{LHH}}\right) /\left(I_{\mathrm{RHH}}+I_{\mathrm{LHH}}\right)
$$

values used to calculate individual partial-wave fractional probabilities shown in figures 5-10. The relative intensity $I$ shown in figures $3-10$, determined by combining individual measurements from the $P_{4}$ detector $\left(I=I_{\|}+2 I_{\perp}\right)$, is proportional, within detector efficiencies and the transmissions of the various optical elements, to the total intensity emitted into $4 \pi$ solid angle [29]. The indicated uncertainties associated with each data point represent $1 \sigma$ counting statistics. In the cases that error bars are not shown, the uncertainties are smaller than the symbol used to represent the datum. The partial-wave intensities sum to give the total intensity.

We begin with some general comments about the partialwave cross sections in figures 5-10. First, the partial-wave partitioning yields resonance features that are now more clearly related to Beutler-Fano shapes. The best two examples of this are narrow dip in the ${ }^{2} \mathrm{D}^{\circ}{ }_{3 / 2}$ data (reaction (3)) at $36.232 \mathrm{eV}$ (figure 7), and the ${ }^{2} \mathrm{~S} 10 \mathrm{p}$ resonance in the ${ }^{2} \mathrm{P}^{\circ}{ }_{1 / 2}$ spectrum (reaction (1); figure 5). In the former case, we see the dip to be due to the addition of a quasi-Lorentzian (very large ' $q$ ') ${ }^{2} S$ p resonance in the $d_{3 / 2}$ partial-wave data with the

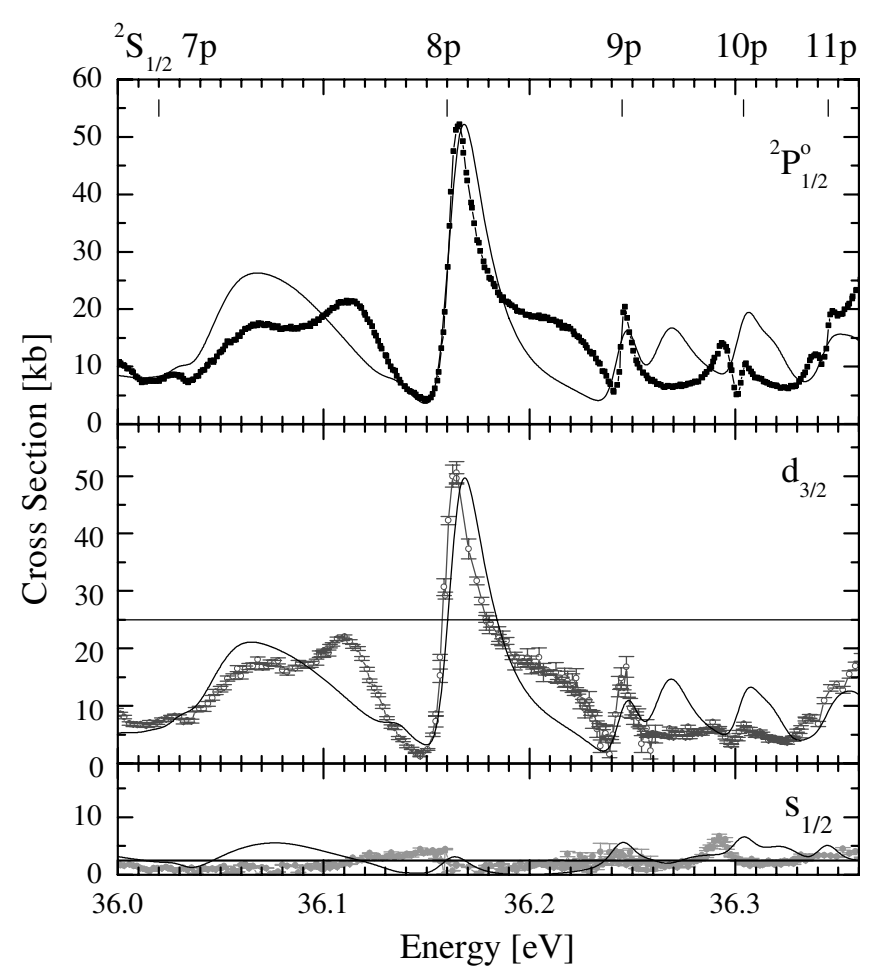

Figure 5. Top panel: relative total fluorescent intensity for reaction (1) $\left(465.8 \mathrm{~nm}-\mathrm{Ar}^{+} 3 \mathrm{p}^{4}\left[{ }^{3} \mathrm{P}\right] 4 \mathrm{p}\left({ }^{2} \mathrm{P}_{1 / 2}\right)\right.$ to $3 \mathrm{p}^{4}\left[{ }^{3} \mathrm{P}\right] 4 \mathrm{~s}\left({ }^{2} \mathrm{P}_{3 / 2}\right)$; black data points and connecting line) normalized to the absolute theoretical calculations of this work (black line) at the cross section maximum near the Ar I $\left(3 s^{2} 3 p^{4}\right)\left[{ }^{1} S\right] 4 s^{2} S$ sp resonance maximum. The resonance designations are the same as in figure 4. Lower panels: intensity due to the individual partial waves (data points and connecting line) with the theoretical calculations overlain (black lines). The sum of the partial-wave intensities equals the total intensity. The experimental resolution and the convolution width of the theoretical calculation are $10 \mathrm{meV}$. Horizontal lines indicate simple $L S$-coupling prediction for the partial-wave cross sections (see section 4.5).

sharp dip in the asymmetric profile of the same resonance in the $\mathrm{d}_{5 / 2}$ data. A weak shoulder in the $\mathrm{d}_{3 / 2}$ data and somewhat more pronounced ridge in the $\mathrm{d}_{5 / 2}$ data, both at $\sim 36.22 \mathrm{eV}$ and both possibly due to the $\mathrm{D}$ resonance, complicates this picture a bit. The oddly shaped 'window' resonance in the total cross section at $36.30 \mathrm{eV}$ (figure 5), suggested in [22] to be caused by the interference of the ${ }^{2} \mathrm{~S} 10 \mathrm{p}$ and $\left[{ }^{1} \mathrm{D}\right] 3 \mathrm{~d}^{2} \mathrm{D} 4 \mathrm{f}$ doubly excited states, is now seen to be simply the summation of the ' $q$ ' $=0$ and ' $q$ ' = infinity ${ }^{2} \mathrm{~S} 10 \mathrm{p}$ resonances in the two partial waves. Having said this, the ${ }^{2} \mathrm{~S} 10 \mathrm{p}$ resonance is obviously interfering with something in at least the $\mathrm{d}_{3 / 2}$ channel. Whether this is a smooth direct-channel background or a broad $\left[{ }^{1} \mathrm{D}\right] 3 \mathrm{~d}{ }^{2} \mathrm{D}$ 4f resonance, as is suggested in [22], is unclear. Indeed, a $4 \mathrm{f}$ resonance would be expected to be extremely broad given its low $n$-value and the significant overlap between $3 \mathrm{~d}$ and $4 \mathrm{f}$ orbitals. We will discuss the former possibility more below.

The second qualitative result of the partial-wave partitioning is the strong variation in their fractional intensities. This is shown qualitatively in table 1 for the two most prominent resonances in the energy range under study, the ${ }^{2} \mathrm{~S} 8 \mathrm{p}$ and $9 \mathrm{p}$ features. In both cases, the $\mathrm{s}$ and $\mathrm{g}$ partial waves 


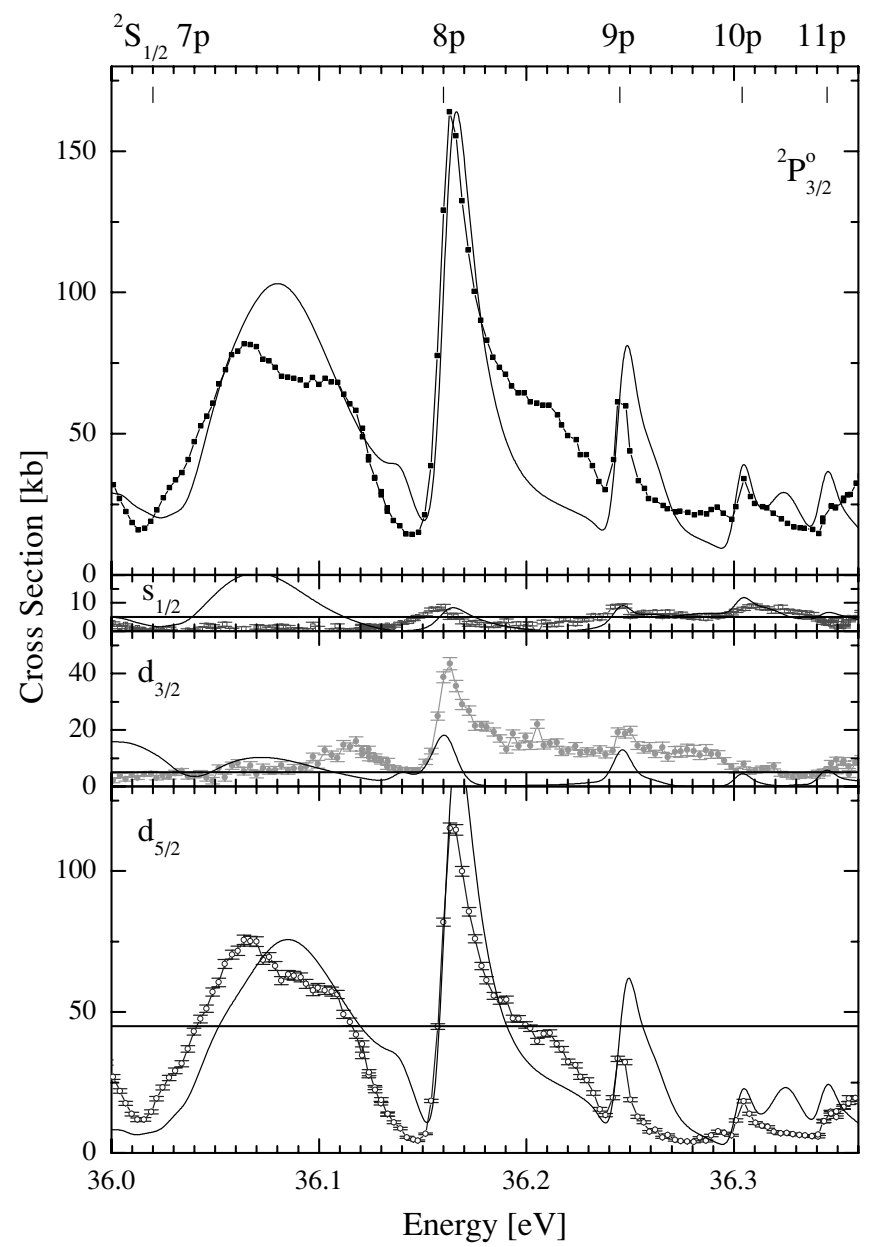

Figure 6. Data equivalent to that in figure 5 except for reaction (2): $476.5 \mathrm{~nm}-\mathrm{Ar}^{+} 3 \mathrm{p}^{4}\left[{ }^{3} \mathrm{P}\right] 4 \mathrm{p}\left({ }^{2} \mathrm{P}_{3 / 2}\right)$ to $3 \mathrm{p}^{4}\left[{ }^{3} \mathrm{P}\right] 4 \mathrm{~s}\left({ }^{2} \mathrm{P}_{1 / 2}\right)$. The experimental resolution and the convolution width of the theoretical calculation are $8 \mathrm{meV}$.

Table 1. Relative strengths of the $\left[{ }^{1} S\right] 4 s^{2} S$ p and $9 p$ doubly excited resonances.

\begin{tabular}{lllll}
\hline & \multicolumn{4}{c}{ Partial waves } \\
\cline { 2 - 5 } State & $\mathrm{s}_{1 / 2}$ & $\mathrm{~d}_{3 / 2}$ & $\mathrm{~d}_{5 / 2}$ & $\mathrm{~g}_{7 / 2}$ \\
\hline \multicolumn{5}{c}{$8 \mathrm{p}$} \\
& & \\
${ }^{2} \mathrm{P}_{1 / 2}$ & 0.05 & 0.95 & & \\
${ }^{2} \mathrm{P}_{3 / 2}$ & 0.05 & 0.25 & 0.7 & \\
${ }^{2} \mathrm{D}_{3 / 2}$ & 0.05 & 0.3 & 0.65 & \\
${ }^{2} \mathrm{D}_{5 / 2}$ & & 0.1 & 0.9 & 0 \\
${ }^{4} \mathrm{P}_{5 / 2}$ & & & & \\
${ }^{4} \mathrm{D}_{5 / 2}$ & & 0.05 & 0.9 & 0.05 \\
& & $9 \mathrm{p}$ & & \\
& & & & \\
${ }^{2} \mathrm{P}_{1 / 2}$ & 0.2 & 0.8 & & \\
${ }^{2} \mathrm{P}_{3 / 2}$ & 0.1 & 0.3 & 0.6 & \\
${ }^{2} \mathrm{D}_{3 / 2}$ & 0.1 & 0.6 & 0.3 & \\
${ }^{2} \mathrm{D}_{5 / 2}$ & & 0.04 & 0.95 & 0.01 \\
${ }^{4} \mathrm{P}_{5 / 2}$ & & 0 & 1 & 0 \\
${ }^{4} \mathrm{D}_{5 / 2}$ & & 0.03 & 0.95 & 0.02 \\
\hline
\end{tabular}

are strongly suppressed. This is expected for the g-waves because of the low outgoing electron energies in this photon

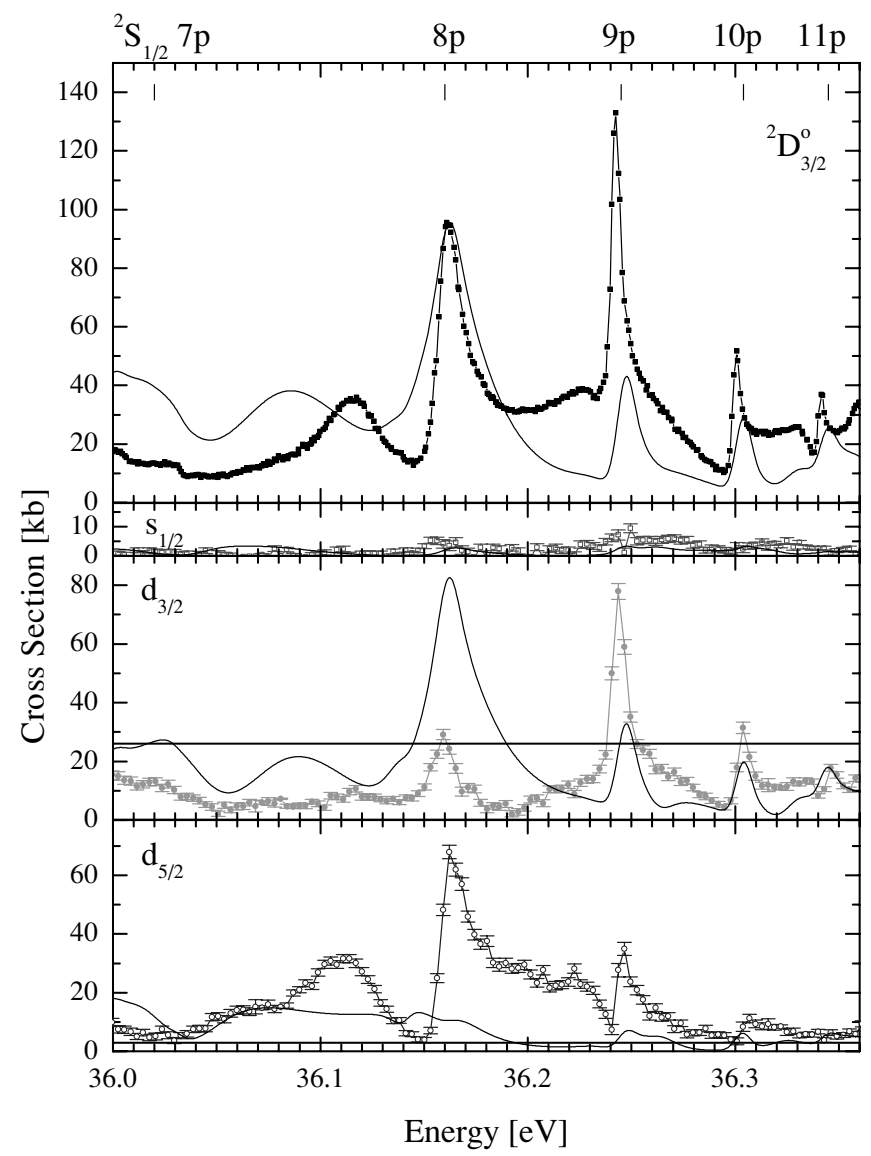

Figure 7. Data equivalent to that in figure 5 except for reaction (3): $472.7 \mathrm{~nm}-\mathrm{Ar}^{+} 3 \mathrm{p}^{4}\left[{ }^{3} \mathrm{P}\right] 4 \mathrm{p}\left({ }^{2} \mathrm{D}_{3 / 2}\right)$ to $3 \mathrm{p}^{4}\left[{ }^{3} \mathrm{P}\right] 4 \mathrm{~s}\left({ }^{2} \mathrm{P}_{3 / 2}\right)$. The experimental resolution and the convolution width of the theoretical calculation are $8 \mathrm{meV}$.

energy range. There does not seem to be any systematic effect for situations which are 'parity favoured' in which the residual ion and the outgoing partial wave have $j$ values differing by one, versus the 'parity unfavoured' case, in which the two $j$ values are the same $[8,18]$. In the case of the $8 p$ resonance, the parity-favored situation prevails with ${ }^{2,4} \mathrm{P}$ residual ions; the opposite occurs with ${ }^{2,4} \mathrm{D}$ ions. For the $9 \mathrm{p}$ resonance, the D-state propensity holds, while the P-state case is mixed. Interestingly, the relative intensities of the $8 p$ and $9 p$-wave intensities flip depending on the $j$ of the residual D-state ions. The partial-wave intensities we observe are consistent with the fluorescence angular distribution measurements made by Mentzel et al [8] in the cases of reactions (2)-(5).

\subsection{Angular momentum coupling considerations}

Some elementary theoretical considerations cast light on the situation presented in table 1 . If we have no spin-orbit coupling in the initial state, and none in the final state except for an infinitesimal amount that produces the spin-orbit splitting of Ar II energy eigenstates, it is possible to enumerate expected constraints on the relative strengths of the different partialwave channels. In this picture, there are two fundamental amplitudes for photoexcitation of the $\mathrm{Ar}^{1} \mathrm{~S}_{0}^{e}$, namely to excited 


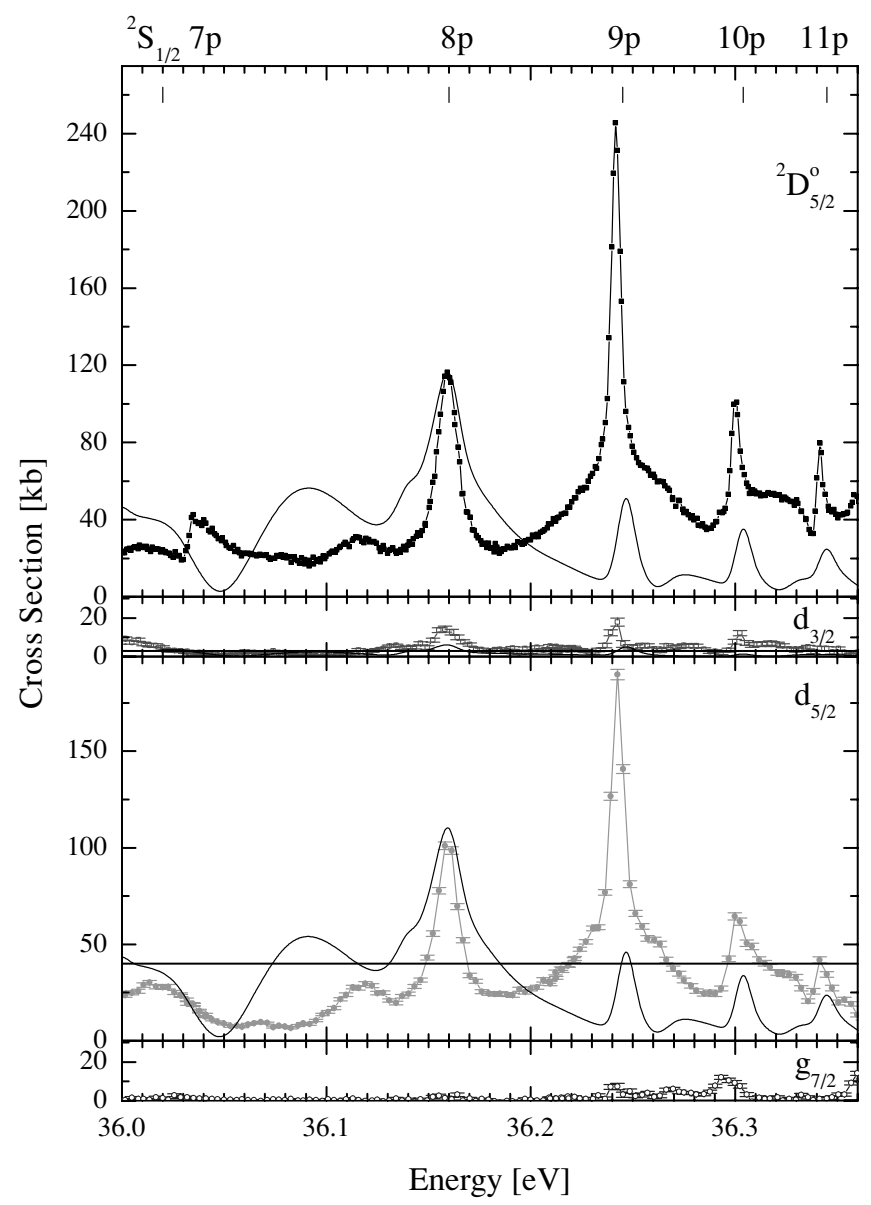

Figure 8. Data equivalent to that in figure 5 except for reaction (4): $488.0 \mathrm{~nm}-\mathrm{Ar}^{+} 3 \mathrm{p}^{4}\left[{ }^{3} \mathrm{P}\right] 4 \mathrm{p}\left({ }^{2} \mathrm{D}_{5 / 2}\right)$ to $3 \mathrm{p}^{4}\left[{ }^{3} \mathrm{P}\right] 4 \mathrm{~s}\left({ }^{2} \mathrm{P}_{3 / 2}\right)$. The experimental resolution and the convolution width of the theoretical calculation are $8 \mathrm{meV}$.

continuum states of two types:

$h v+\operatorname{Ar}\left[{ }^{1} \mathrm{~S}_{0}^{e}\right] \rightarrow\left\{\operatorname{Ar}^{+}\left[{ }^{2 S_{c}+1} L_{c J_{c}}\right]+e[\varepsilon s\right.$ or $\left.\varepsilon d]\right\}\left({ }^{1} \mathrm{P}_{1}^{o}\right)$.

In other words, the photoexcitation produces in $L S$-coupling only three alternative excited complexes having ${ }^{1} \mathrm{P}_{1}^{\mathrm{o}}$ character, and whose relative strengths depend on the dynamics of the photoionization process. These three reduced dipole matrix amplitudes have the following structure: $C\left({ }^{2} L_{c} \varepsilon l\right)=$ $\left\langle\left\{\left(L_{c} l\right) L_{\mathrm{tot}}\left(S_{c} s\right) S_{\mathrm{tot}}\right\} J_{\mathrm{tot}}=1\left\|r^{(1)}\right\|(00) J_{0}=0\right\rangle$, where

$$
\begin{aligned}
& C_{\mathrm{Ps}}=C\left({ }^{2} \mathrm{P}^{\mathrm{o}} \varepsilon s\right) \\
& =\left\langle\left\{(10) 1\left(\frac{1}{2} \frac{1}{2}\right) 0\right\} 1=1\left\|r^{(1)}\right\|(00) J_{0}=0\right\rangle,
\end{aligned}
$$

$$
\begin{aligned}
C_{\mathrm{Pd}} & =C\left({ }^{2} \mathrm{P}^{\mathrm{o}}, \varepsilon d\right) \\
& =\left\langle\left\{(12) 1\left(\frac{1}{2} \frac{1}{2}\right) 0\right\} 1=1\left\|r^{(1)}\right\|(00) J_{0}=0\right\rangle,
\end{aligned}
$$

and

$$
\begin{aligned}
& C_{\mathrm{Dd}}=C\left({ }^{2} \mathrm{D}^{\mathrm{o}} \varepsilon d\right) \\
& =\left\langle\left\{(22) 1\left(\frac{1}{2} \frac{1}{2}\right) 0\right\} 1=1\left\|r^{(1)}\right\|(00) J_{0}=0\right\rangle .
\end{aligned}
$$

In the absence of resonance physics, one can determine the partial-wave amplitudes in terms of these three $C$ amplitudes

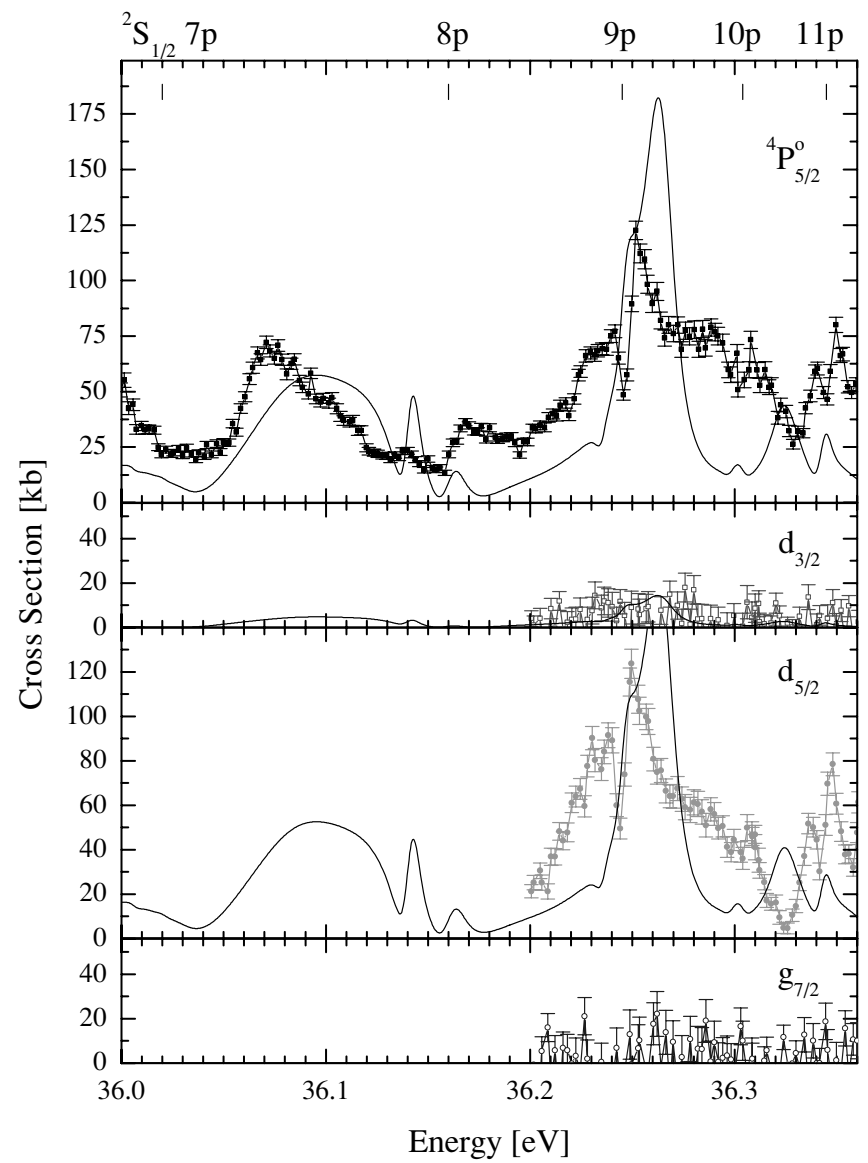

Figure 9. Data equivalent to that in figure 5 except for reaction (5): $480.6 \mathrm{~nm}-\mathrm{Ar}^{+} 3 \mathrm{p}^{4}\left[{ }^{3} \mathrm{P}\right] 4 \mathrm{p}\left({ }^{4} \mathrm{P}_{5 / 2}\right)$ to $3 \mathrm{p}^{4}\left[{ }^{3} \mathrm{P}\right] 4 \mathrm{~s}\left({ }^{4} \mathrm{P}_{5 / 2}\right)$. The experimental resolution and convolution width of theory are $5 \mathrm{meV}$. Experimental data are normalized to the theoretical cross section at the experimental data maximum near the $9 p$ resonance.

by carrying out a Wigner-Racah $L S$-jj recoupling, i.e. by transforming from the $\left|\left\{\left(L_{c} l\right) L_{\mathrm{tot}}\left(S_{c} s\right) S_{\mathrm{tot}}\right\} J_{\mathrm{tot}}=1, M_{\mathrm{tot}}=1\right\rangle$ representation to the $\left|\left\{\left(L_{c} S_{c}\right) J(l s) j_{e}\right\} J_{\mathrm{tot}}=1, M_{\mathrm{tot}}=1\right\rangle$ representation, using the Wigner $9 j$ coefficient

$$
\begin{aligned}
& \left.\left\langle\left(L_{c} l\right) L_{\mathrm{tot}}\left(S_{c} s\right) S_{\mathrm{tot}}\right|\left(L_{c} S_{c}\right) J(l s) j_{e}\right)^{\left(J_{\text {tot }}\right)} \\
& =\left[L_{\mathrm{tot}}, S_{\mathrm{tot}}, J, j_{e}\right]^{1 / 2}\left\{\begin{array}{ccc}
L_{c} & l & L_{\mathrm{tot}} \\
S_{c} & s & S_{\mathrm{tot}} \\
J & j_{e} & J_{\mathrm{tot}}
\end{array}\right\},
\end{aligned}
$$

where the notation $[x, y, \ldots]^{1 / 2}$ stands for $[(2 x+1)(2 y+1) \ldots]^{1 / 2}$. This results in the following expressions:

$$
\begin{gathered}
C\left({ }^{2} \mathrm{P}_{J}^{\mathrm{o}} \varepsilon s_{\frac{1}{2}}\right)=C\left({ }^{2} \mathrm{P}^{\mathrm{o}} \varepsilon s\right)\left\langle(10) 1\left(\frac{1}{2} \frac{1}{2}\right) 0 \mid\left(1 \frac{1}{2}\right) J\left(0 \frac{1}{2}\right) \frac{1}{2}\right\rangle^{(1)} \\
=\left\{\begin{array}{l}
-\sqrt{\frac{1}{3}}, J=\frac{1}{2}, j_{e}=\frac{1}{2} \\
\sqrt{\frac{2}{3}}, J=\frac{3}{2}, j_{e}=\frac{1}{2},
\end{array}\right.
\end{gathered}
$$




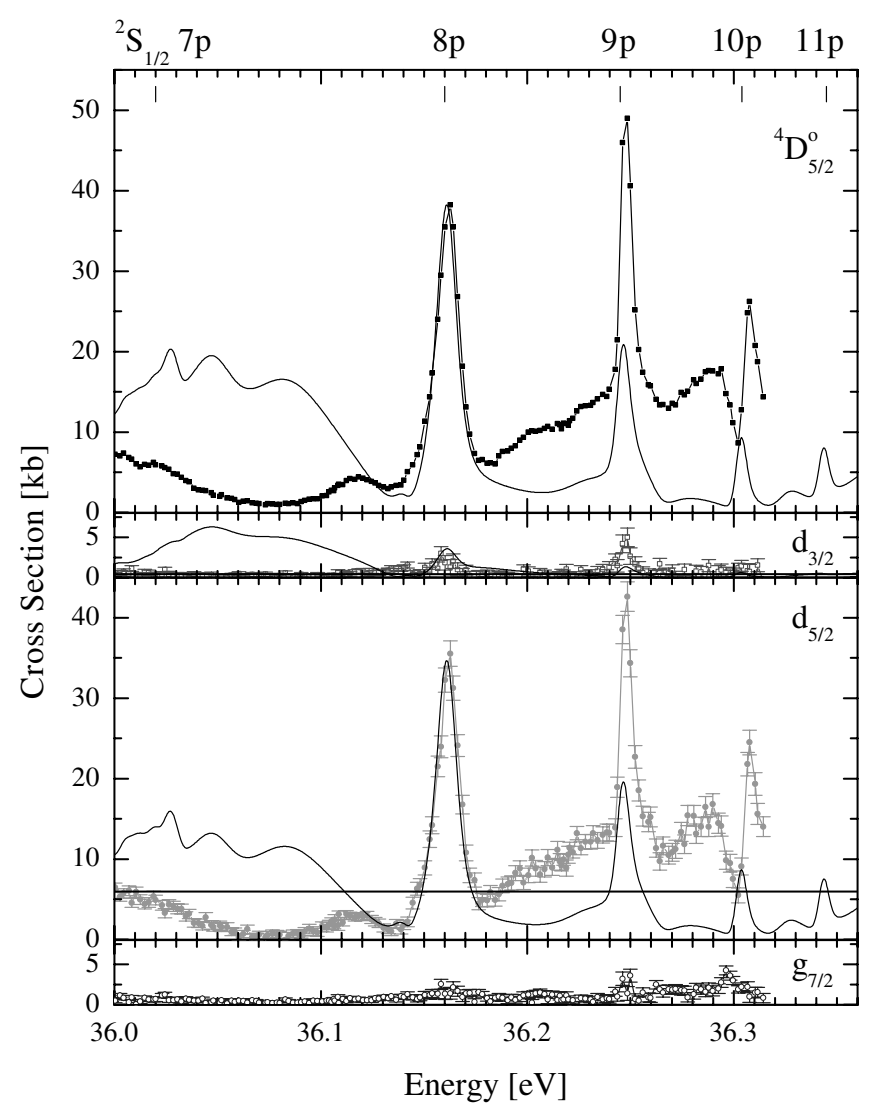

Figure 10. Data equivalent to that in figure 5 except for reaction (6): $514.5 \mathrm{~nm}-\mathrm{Ar}^{+} 3 \mathrm{p}^{4}\left[{ }^{3} \mathrm{P}\right] 4 \mathrm{p}\left({ }^{4} \mathrm{D}_{5 / 2}\right)$ to $3 \mathrm{p}^{4}\left[{ }^{3} \mathrm{P}\right] 4 \mathrm{~s}\left({ }^{2} \mathrm{P}_{3 / 2}\right)$. The experimental resolution and the convolution width of the theoretical calculation are $5 \mathrm{meV}$.

$$
\begin{gathered}
C\left({ }^{2} \mathrm{P}_{J}^{\mathrm{o}} \varepsilon d_{j_{e}}\right)=C\left({ }^{2} \mathrm{P}^{\mathrm{o}} \varepsilon d\right)\left\langle(12) 1\left(\frac{1}{2} \frac{1}{2}\right) 0 \mid\left(1 \frac{1}{2}\right) J\left(2 \frac{1}{2}\right) j_{e}\right\rangle^{(1)} \\
=\left\{\begin{array}{l}
\sqrt{\frac{1}{3}}, J=\frac{1}{2}, j_{e}=\frac{3}{2} \\
\sqrt{\frac{1}{15}}, J=\frac{3}{2}, j_{e}=\frac{3}{2} \\
\sqrt{\frac{3}{5}}, J=\frac{3}{2}, j_{e}=\frac{5}{2}
\end{array}\right.
\end{gathered}
$$

and

$$
\begin{aligned}
& C\left({ }^{2} \mathrm{D}_{J}^{\mathrm{o}} \varepsilon d_{j_{e}}\right)=C\left({ }^{2} \mathrm{D}^{\mathrm{o}} \varepsilon d\right)\left\langle(22) 1\left(\frac{1}{2} \frac{1}{2}\right) 0 \mid\left(2 \frac{1}{2}\right) J\left(2 \frac{1}{2}\right) j_{e}\right\rangle^{(1)} \\
& =\left\{\begin{array}{l}
\frac{3}{5}, J=\frac{3}{2}, j_{e}=\frac{3}{2} \\
-\frac{1}{5}, J=\frac{3}{2}, j_{e}=\frac{5}{2} \\
\frac{1}{5}, J=\frac{5}{2}, j_{e}=\frac{3}{2} \\
\sqrt{\frac{14}{25}}, J=\frac{5}{2}, j_{e}=\frac{5}{2}
\end{array}\right.
\end{aligned}
$$

Thus, in a strict $L S$-coupling approximation, and neglecting $g$-waves, these are the only channels that would be populated in an argon photoionization experiment in this energy range. The pure- $L S$ approximation would lead to the following relationships among the branching ratios for the different channels. The numerical values listed have been obtained using estimated values for the three $L S$ probabilities, taken to equal $\left|C_{\mathrm{Ps}}\right|^{2} \cong 7.5 \mathrm{~kb},\left|C_{\mathrm{Pd}}\right|^{2} \cong 75 \mathrm{~kb}$, and $\left|C_{\mathrm{Dd}}\right|^{2} \cong$ $71 \mathrm{~kb}$ :

$$
\begin{gathered}
\sigma\left[{ }^{2} \mathrm{P}_{\frac{1}{2}}^{\mathrm{o}} \varepsilon s_{\frac{1}{2}}\right]=\frac{1}{3}\left|C_{\mathrm{Ps}}\right|^{2} \rightarrow 2.5 \mathrm{~kb}, \\
\sigma\left[{ }^{2} \mathrm{P}_{\frac{1}{2}}^{\mathrm{o}} \varepsilon d_{\frac{3}{2}}\right]=\frac{1}{3}\left|C_{\mathrm{Pd}}\right|^{2} \rightarrow 25 \mathrm{~kb}, \\
\sigma\left[{ }^{2} \mathrm{P}_{\frac{3}{2}}^{\mathrm{o}} \varepsilon s_{\frac{1}{2}}\right]=\frac{2}{3}\left|C_{\mathrm{Ps}}\right|^{2} \rightarrow 5.0 \mathrm{~kb}, \\
\sigma\left[{ }^{2} \mathrm{P}_{\frac{3}{2}}^{\mathrm{o}} \varepsilon d_{\frac{3}{2}}\right]=\frac{1}{15}\left|C_{\mathrm{Pd}}\right|^{2} \rightarrow 5.0 \mathrm{~kb}, \\
\sigma\left[{ }^{2} \mathrm{P}_{\frac{3}{2}}^{\mathrm{o}} \varepsilon d_{\frac{5}{2}}\right]=\frac{3}{5}\left|C_{\mathrm{Pd}}\right|^{2} \rightarrow 45 \mathrm{~kb}, \\
\sigma\left[{ }^{2} \mathrm{D}_{\frac{3}{2}}^{\mathrm{o}} \varepsilon d_{\frac{3}{2}}\right]=\frac{9}{25}\left|C_{\mathrm{Dd}}\right|^{2} \rightarrow 26 \mathrm{~kb}, \\
\sigma\left[{ }^{2} \mathrm{D}_{\frac{3}{2}}^{\mathrm{o}} \varepsilon d_{\frac{5}{2}}\right]=\frac{1}{25}\left|C_{\mathrm{Dd}}\right|^{2} \rightarrow 2.9 \mathrm{~kb}, \\
\sigma\left[{ }^{2} \mathrm{D}_{\frac{5}{2}}^{\mathrm{o}} \varepsilon d_{\frac{3}{2}}\right]=\frac{1}{25}\left|C_{\mathrm{Dd}}\right|^{2} \rightarrow 2.9 \mathrm{~kb}, \\
\sigma\left[{ }^{2} \mathrm{D}_{\frac{5}{2}}^{\mathrm{o}} \varepsilon d_{\frac{5}{2}}\right]=\frac{14}{25}\left|C_{\mathrm{Dd}}\right|^{2} \rightarrow 40 \mathrm{~kb} .
\end{gathered}
$$

These values are indicated for the appropriate partial-wave data in figures 5-8.

Next, if we consider the quartet states, it is of course immediately clear that these could not have any amplitude at all in strict $L S$-coupling. However, the ${ }^{4} \mathrm{P}_{5 / 2}^{\mathrm{o}}$ ' level of $\mathrm{Ar}$ II has a small amount of doublet character, with the largest contribution by an order of magnitude being the ${ }^{2} \mathrm{D}_{5 / 2}^{\mathrm{o}}$ state, with $0.1 \%$ total weight. Thus

$$
\begin{aligned}
& \sigma\left[{ }^{4} \mathrm{P}_{\frac{5}{2}}^{\mathrm{o}} \varepsilon d_{\frac{3}{2}}\right]=\frac{1}{25}\left|C_{\mathrm{Dd}}\right|^{2} \rightarrow 0.003 \mathrm{~kb}, \\
& \sigma\left[{ }^{4} \mathrm{P}_{\frac{5}{2}}^{\mathrm{o}} \varepsilon d_{\frac{5}{2}}\right]=\frac{14}{25}\left|C_{\mathrm{Dd}}\right|^{2} \rightarrow 0.040 \mathrm{~kb},
\end{aligned}
$$

and

$$
\sigma\left[{ }^{4} \mathrm{P}_{\frac{5}{2}}^{\mathrm{o}} \varepsilon g_{\frac{7}{2}}\right]=0 .
$$

Similarly [14], the " ${ }^{4} \mathrm{D}_{5 / 2}^{\mathrm{o}}$ ' level of $\mathrm{Ar}$ II has a nonnegligible amount of doublet character, predominantly through a state of ${ }^{2} \mathrm{D}_{5 / 2}^{\mathrm{o}}$ character with weight $15 \%$, giving the following expectation for a direct, nonresonant photoionization process:

$$
\begin{gathered}
\sigma\left[{ }^{4} \mathrm{D}_{\frac{5}{2}}^{\mathrm{o}} \varepsilon d_{\frac{3}{2}}\right]=\frac{1}{25}\left|C_{\mathrm{Dd}}\right|^{2} \rightarrow 0.44 \mathrm{~kb}, \\
\sigma\left[{ }^{4} \mathrm{D}_{\frac{5}{2}}^{\mathrm{o}} \varepsilon d_{\frac{5}{2}}\right]=\frac{14}{25}\left|C_{\mathrm{Dd}}\right|^{2} \rightarrow 6.0 \mathrm{~kb},
\end{gathered}
$$

and

$$
\sigma\left[{ }^{4} \mathrm{D}_{\frac{5}{2}}^{\mathrm{o}} \varepsilon g_{\frac{7}{2}}\right]=0
$$

(These 'quartet' expected values are indicated in figures 9 and 10.) Thus if the initial photoabsorption step still excites only the ${ }^{1} \mathrm{P}_{1}^{\mathrm{o}}$ complex $\left(\mathrm{Ar}^{+}+\mathrm{e}^{-}\right)$, the partial weights for the two $L S$-forbidden ionic states ' ${ }^{4} \mathrm{P}_{5 / 2}^{\mathrm{o}}$ ' and ${ }^{4} \mathrm{D}_{5 / 2}^{\mathrm{o}}$ ' should have the same partial-wave amplitudes as listed above for ${ }^{2} \mathrm{D}_{5 / 2}^{\mathrm{o}}$ level, except that they must be multiplied by the respective ${ }^{2} \mathrm{D}_{5 / 2}^{0}$ admixture coefficients from [14]. Inspection of the data in table 1 and in figures 5-10 shows general consistency with this expected pattern for the ${ }^{4} \mathrm{D}_{5 / 2}^{\mathrm{o}}$ ' partial cross sections. However, this argument underpredicts the ${ }^{4} \mathrm{P}_{5 / 2}^{0}$ ' partialwave cross sections by 2-3 orders of magnitude. This discrepancy might be an indication that the theoretical analysis of [14] grossly underestimates the amount of ${ }^{2} \mathrm{D}_{5 / 2}^{\mathrm{o}}$ character 
in the ' ${ }^{4} \mathrm{P}_{5 / 2}^{\mathrm{o}}$ ' state of Ar II. The only realistic alternative interpretation could be that doubly excited resonances with significant triplet character are being predominantly excited in this energy range. For direct photoionization of Ar, it is well known that continuum $\mathrm{d}$ electrons are ejected with a probability around one order of magnitude stronger than $\mathrm{s}$ electrons [30]; this dominance of d-wave photoionization appears to be borne out for both resonances listed in table 1, although the dominance is less clear for the $9 p$ resonance where it is only $4: 1$ compared to $19: 1$ for the $8 \mathrm{p}$ resonance at least in the production of ${ }^{2} \mathrm{P}_{1 / 2}^{\mathrm{o}}$ ions. On the other hand, for the production of ${ }^{2} \mathrm{P}_{3 / 2}^{\mathrm{o}}$ ions, the d:s ratio is closer to the 'expected' value, namely 9:1.

The branching ratio $\sigma\left[{ }^{2} \mathrm{P}_{3 / 2}^{\mathrm{o}} \varepsilon \mathrm{d}_{3 / 2}\right]: \quad \sigma\left[{ }^{2} \mathrm{P}_{3 / 2}^{\mathrm{o}} \varepsilon \mathrm{d}_{5 / 2}\right]$ is predicted by this simple recoupling argument to be 1:9 whereas the experimental result for $8 p$ is $1: 2.8$ and for $9 p$ it is $1: 2$, both representing a major departure from this simple theoretical expectation, which presumably reflects the breakdown of $L S$ coupling in the doubly excited resonant state. On the other hand, if one refers to the cross sections expected from this analysis and indicated for the respective partial waves in figures 5-10, it suggests that the theoretical expectation is not too far off for the average branching ratio of these two channels across the energy range depicted. This is because the branching ratios implied by the recoupling argument above should be approximately applicable to either direct photoionization or to autoionization from a resonance of predominantly ${ }^{1} \mathrm{P}^{\circ}$ character.

\subsection{Other manifestations of spin-orbit coupling}

We turn finally to the question of what qualitative physical information we can learn from the partial-wave cross sections with regard to spin-orbit coupling in the photo-ejection process. It is important to note that even at this level of detail, the partial-wave spectra we observe are not associated with single final channels, because we do not determine their individual magnetic quantum numbers. To determine the $\left(j_{e}, m_{j e}\right)$ cross sections would require electron-photon coincidence measurements which, with the current generation of synchrotron light sources, are not practicable with this level of energy resolution and polarization analysis. We can thus not extract quantitative information about Beutler-Fano shape parameters. (This is why we use the notation ' $q$ ' instead of $\mathrm{q}$ to refer to the resonance profiles in this paper.) Our results do, however, provide enough detail to allow us to reasonably speculate about the role played by various angular momentum coupling mechanisms in these collisions.

We begin by identifying four distinct mechanisms (figure 11) for the magnetic interactions, ignoring the usually negligible spin-spin interactions: (i) internal spin-orbit coupling between the electrons and the nucleus of the residual ion, which leads to the observation of spin polarization of the photoelectron when the fine structure of the ion is resolved [31], and which allows us to spectroscopically resolve fluorescence from different fine-structure states in these systems; (ii) internal spin-orbit coupling in a resonant autoionizing state of doubly excited, neutral atoms, (i)

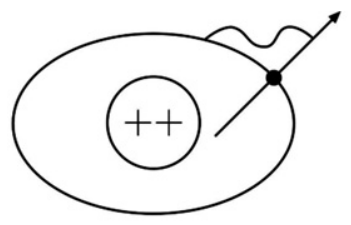

(ii)

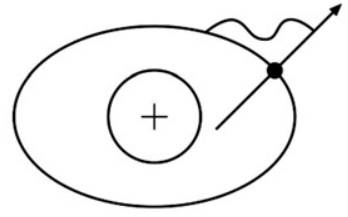

(iii)

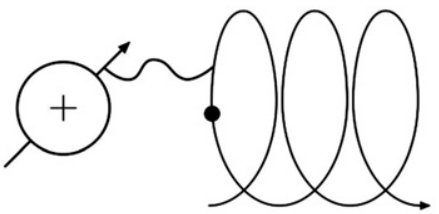

(iv)

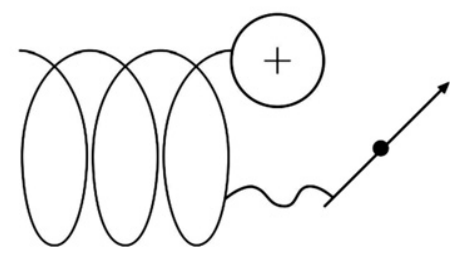

Figure 11. Spin-orbit coupling mechanisms in photoionization/ excitation (see the text).

(iii) interaction of the overall magnetic moment of the residual ion with the magnetic field caused by the motion of the photoelectron in the reference frame centred on the ion, (i.e., the spin-other-orbit and orbit-orbit magnetic terms in the Hamiltonian) and (iv) the Fano effect [32], which is the phaseshift difference associated with the photoelectron's own spin-orbit interaction (this last case is analogous to Mott scattering). To our knowledge, case (iii) has not been discussed in the literature in the context of photoionization.

We concentrate on the last three mechanisms, since the first is evident simply by resolving fine-structure components in the fluorescence we observe and in the differences between intensity or partial-wave spectra with the same spin multiplicity and $L$-value, but with different $\mathrm{J}$, as discussed above (figure 4). We are particularly interested in what can be learned from interference effects manifested in the shapes of the resonance profiles. The ${ }^{2} \mathrm{~S} n \mathrm{p}$ resonances are unique in this regard because at the partial-wave level they exhibit unambiguous asymmetry. In the two other sets of partial-wave data we have published in preliminary reports for excitation of the $\left(3 p^{4}\left[{ }^{3} \mathrm{P}\right] 4 \mathrm{p}\right){ }^{2} \mathrm{P}_{1 / 2}^{\circ}$ and $\left(3 \mathrm{p}^{4}\left[{ }^{3} \mathrm{P}\right] 4 \mathrm{p}\right){ }^{2} \mathrm{P}^{\circ}{ }_{3 / 2}$ states (reactions (1) and (2) respectively), none of the resonances are asymmetric except for the $4 \mathrm{~s}{ }^{2} \mathrm{~S}$ ones in the energy range between 36.0 and $36.4 \mathrm{eV}$. In the absence of any clear evidence for a direct-process background, what we can say is that the asymmetric profiles constitute clear evidence for interference between two different autoionizing resonances leading to the same ionic state. To our knowledge, no other 
data showing effects of this type have identified the quantum numbers of the final channel.

The best example of such interference is seen in the reaction (3) ${ }^{2} \mathrm{D}^{\circ}{ }_{3 / 2}$ data for the $8 \mathrm{p}$ and $9 \mathrm{p}{ }^{2} \mathrm{~S}$ resonances (figure 7 ), which are symmetric in the $d_{3 / 2}$ wave, but are asymmetric in the $d_{5 / 2}$ case. This is due to the differences in the phase between these resonances and broad underlying resonances in the $\mathrm{d}_{5 / 2}$ spectrum associated with the $\mathrm{C}$ or $\mathrm{D}$ states or other, as yet unidentified resonances. Interference with broad resonances is less evident in the $\mathrm{d}_{3 / 2}$ case, which is comprised of much more symmetric features, although the 'shoulder' on the ${ }^{2} \mathrm{~S} 9 \mathrm{p}$ resonance in the $\mathrm{d}_{3 / 2}$ data may indicate contributions from the D resonance. Interestingly, similar behaviour is not seen (at least as obviously) in the reaction (4) ${ }^{2} \mathrm{D}^{\circ}{ }_{5 / 2}$ fine-structure D-twin (figure 8). If a direct continuum background could be unambiguously identified for the two reaction (3) d-waves, the difference in resonance shapes would be direct evidence for mechanism (iv), the Fano effect, in this system. Under this assumption, the 'half-collision' scattering phase [33] of the direct process is obviously different for the two partial waves; in the $d_{3 / 2}$ process, we see an almost pure Lorentzian with very high 'q', whereas in the $\mathrm{d}_{5 / 2}$ case, ' $\mathrm{q}$ ' $\sim 1$. Since resonance phases always change by $\pi$ radians across their widths, the corresponding absolute phase difference between the two $d$ partial-wave amplitudes is $\sim \pi / 2$ radians at the energy where the two reactions enter resonance.

Similar phase shifting for different $l$ partial waves is evident in the $\mathrm{s}_{1 / 2}$ and $\mathrm{d}_{3 / 2}$ data for reaction $(1)^{2} \mathrm{P}^{\circ}{ }_{1 / 2}$ excitation between 36.27 and $36.325 \mathrm{eV}$ (figure 5). Since, apart from the ${ }^{2} \mathrm{~S} 10 \mathrm{p}$ resonance, there are no resonances in this region that have been identified previously or that are evident from the current data, and since the ${ }^{2} \mathrm{~S} 10 \mathrm{p}$ resonance width is significantly smaller than its separation from the $9 p$ and $11 p$ terms in the same series, it is not unreasonable to take the background in this section of the spectrum as being due to the direct photoionization of the target. This gives particularly clean evidence for how the direct process phase shift changes with $l$, but does not provide evidence for a Fano effect.

If one can infer a direct background, mechanism (iii) becomes relevant as well. We consider next the quartet $\left(3 \mathrm{p}^{4}\left[{ }^{3} \mathrm{P}\right] 4 \mathrm{p}\right){ }^{4} \mathrm{P}^{\circ}{ }_{5 / 2}$ and $\left(3 \mathrm{p}^{4}\left[{ }^{3} \mathrm{P}\right] 4 \mathrm{p}\right){ }^{4} \mathrm{D}^{\circ}{ }_{5 / 2}$ states (reactions (5) and (6); figures 9 and 10 respectively). Reaction (5) has been investigated recently by Mentzel et al as well [8], and has been considered theoretically in separate calculations by van der Hart and Greene [24]. The formation of such states represents an unambiguous experimental signature of relativistic interactions because it requires a spin nonconserving interaction. Pure dipole transitions conserve spin in the non-relativistic limit and can only lead to an overall excited singlet state in the continuum having $J_{\text {total }}=1$ for a target atom with $J_{\text {target }}=0$ initially. Thus, spin-conserving interactions can only form doublet states of Ar II. While the presence of relativistic effects has been previously observed in this system by other means [5, 8-12], the quartet states provide a particularly direct way to study their dynamical nature, as we will now show.

Van der Hart and Greene [24] have calculated and replicated some of the cross section and alignment features of quartet states measured in [8] by including in their description of the doubly excited $\mathrm{Ar}^{* *}$ wavefunction the triplet, quintet, and ${ }^{1} \mathrm{P}_{1}$ states. This mixing of the triplets and quintets with the radiatively populated ${ }^{1} \mathrm{P}_{1}$ state is made possible by the spin-orbit interaction within the intermediary $\mathrm{Ar}^{* *}$, i.e. through mechanism (ii) in our list above. The formation and subsequent decay by autoionization of an $\mathrm{Ar}^{* *}$ triplet (or quintet) component can lead to quartet states of excited $\mathrm{Ar}$ II. Because there is no inclusion of amplitudes for a direct process yielding a continuum electron that involves a spinorbit interaction in this description, there can be no interference effects that lead to asymmetric Beutler-Fano profiles in the energy dependence of the quartet-state cross sections. Indeed, the measurements of [8] give no indication of anything other than pure Lorentzian resonance profiles, suggesting that direct processes to the continuum have small amplitudes.

Our results for the ${ }^{4} \mathrm{P}$ ionic states, taken with significantly higher resolution than the data of [8] do not challenge this picture. However, we now see clear evidence for interference of the ${ }^{2} \mathrm{~S} 9 \mathrm{p}$ narrow resonance with a much broader resonance which we attribute to the $\left[{ }^{1} \mathrm{D}\right] 4 \mathrm{p}^{2} \mathrm{D}_{3 / 2} 6$ s excitation labelled $\mathrm{D}$. Since the $4 p$ and $6 s$ electrons interact strongly, this state will decay rapidly, leading to a broad resonance. Reaction (6) ${ }^{4} \mathrm{D}$ data are qualitatively different, however. There appears here to be either a linearly increasing background due to a direct process starting at $\sim 36.12 \mathrm{eV}$, or the low-energy half of a very broad $(\mathrm{FWHM} \sim 0.4 \mathrm{eV}$ ) centered at $\sim 36.3 \mathrm{eV}$ upon which the ${ }^{2} \mathrm{~S} 8 \mathrm{p}, 9 \mathrm{p}$ and $10 \mathrm{p}$ resonances are superimposed. (We assume that the small symmetric feature at $36.118 \mathrm{eV}$ is due to the B resonance.) Whatever the nature of the background, the ' $q$ ' of the three ${ }^{2} \mathrm{~S}$ resonances changes from being large and positive for the $8 \mathrm{p}$ feature, smaller but still positive for the $9 \mathrm{p}$, and smaller still in magnitude but negative for the $10 \mathrm{p}$ resonance. This phenomenon has been referred to as 'q-reversal' by Connerade and co-workers [2, 34, 35] for the case of a Rydberg series superimposed on a broad resonance. If the asymmetric shapes of the $9 p$ and $10 p$ peaks are due to interference with a broad, unidentified resonance, the situation is similar to that of the ${ }^{4} \mathrm{P}$ spectrum.

We note, in this context, that a possible second example of q-reversal might be the change of the $\left[{ }^{1} \mathrm{~S}\right] 4 \mathrm{~s}^{2} \mathrm{~S}_{1 / 2} n \mathrm{p}$ resonances, labelled as Rydberg series 4 in figure 3, from the prominent peaks of the $n=8$ and 9 terms to window resonances in the case of $n=6$ and 7. This would imply a very broad resonance, centered at $\sim 36.1 \mathrm{eV}$, with a full width of perhaps $1 \mathrm{eV}$ that interferes with this series. Having said this, the distinction between a resonant interaction and direct photoionization becomes blurred for 'resonances' this broad. The decay time of such a compound would be $\sim 1$ fs, the time required for a prompt photoelectron to leave the interaction region!

Since we have no experimental evidence for such a broad resonance, however, the possibility exists that the background is, in fact, due to a direct process. The very existence of a direct ionization leading to a well- $L S$ coupled quartet state means that the spin-other-orbit coupling mechanism (iii) is operative. This mechanism has not been observed previously and has not, to our knowledge, been postulated prior to this. 
The picture is complicated, however, by the fact that the ${ }^{4} \mathrm{D}$ state populated in reaction (6) is not a pure quartet, unlike the ${ }^{4} \mathrm{P}$ state [14]. This means that a pure spin doublet state (not requiring a spin-flip) can be formed in either a direct or resonant process, and subsequently turn into a 'quartet' state through either mechanism (i) or (ii). Thus, it is not possible to obtain unambiguous evidence for spin-other orbit coupling in reaction (6) from these data.

\section{Conclusions}

By combining high-resolution spectroscopy of photoionization satellite fluorescence in combination with complete polarization analysis of this fluorescence, we have studied the photoionization excitation of $\mathrm{Ar}$ in unprecedented detail. We have obtained unique information about the role played by resonances in the ionization process. Extraction of partial-wave cross sections simplifies the fluorescent excitation spectra, yielding resonance profiles more characteristic of Beutler-Fano interference than those seen in the total intensity spectra. This in turn helps in the identification of the importance of specific resonances in the ionization/excitation process. These roles are shown to be quite sensitive, at this level of detail, to the fine-structure state of the residual ion; individual partial waves may exhibit unique interference effects that can be obscured in the total cross section. Relative cross sections determined either from fluorescent intensity or electron emission measurements may not exhibit a meaningful energy profile, in the sense that one can extract actual Beutler-Fano parameters, unless it can be shown that only one partial wave contributes to the cross section. Our data, however, show unambiguously that the various partial waves of the outgoing electron in a given reaction leading to a particular final state have different phase relationships with underlying broad (i.e., slowly varying) resonant or direct channels.

These data provide the first evidence for the interference between amplitudes having different pathways in the doubly excited electron continuum that leads to the photo-production of excited ions. Evidence for the Fano effect in Ar, as well as a previously unidentified mechanism: spin-other-orbit coupling in the continuum production of quartet states, is problematic, being complicated by the possibility of interference with resonances in the same energy regimes. However, it is important to note that such resonances have not been identified in this region-we only speculate as to their existence. Until more experimental or theoretical work demonstrates the existence or lack of such resonances unambiguously, the question of the importance of these spin-orbit mechanisms will remain open. It is hoped that these measurements will provide benchmark data for theories of photoionization which include the various effects of spin-orbit coupling in more detail.

\section{Acknowledgements}

This work is supported by the National Science Foundation through Grants PHY-9731869, -0099363 and -0321055. The contributions of $\mathrm{CHG}$ and $\mathrm{H}$ vdH have been supported in part by the Department of Energy, Office of Science. We thank ST Manson and CD Lin for stimulating discussions and the staff of the ALS for scientific support.

\section{References}

[1] Madden R P, Ederer D L and Codling K 1969 Phys. Rev. 177136

[2] Baig M A, Ahmad S, Connerade J-P, Dussa W and Hormes J 1992 Phys. Rev. A 457963

[3] Hall R I, Avaldi L, Dawber G, Rutter P M, MacDonald M A and King G C 1989 J. Phys. B: At. Mol. Opt. Phys. 223205

[4] Wills A A, Cafolla A A, Currell F J, Comer J, Svensson A and MacDonald M A 1989 J. Phys. B: At. Mol. Opt. Phys. 223217

[5] Canton-Rogan S E, Wills A A, Gorczyca T W, Wiedenhoeft M, Nayandin O, Liu C-N and Berrah N 2000 Phys. Rev. Lett. 853113

[6] Canton S E, Wills A A, Gorczyca T W, Sokell E, Bozek J D, Turri G, Wiedenhoeft M, Feng X and Berrah N 2003 J. Phys. B: At. Mol. Opt. Phys. $36 \mathrm{~L} 181$

[7] Samson J A R, Lee E-M and Chung Y 1993 J. Electron Spectrosc. Relat. Phenom. 6675

[8] Mentzel G et al 1998 J. Phys. B: At. Mol. Opt. Phys. 31227

[9] Yenen O, McLaughlin K W and Jaecks D H 1997 Phys. Rev. Lett. 795222

[10] McLaughlin K W, Yenen O and Jaecks D H 1998 Phys. Rev. Lett. 81289

[11] Yenen O, McLaughlin K W, Jaecks D H, Sant'Anna M M and Seddon E A 2001 Phys. Rev. Lett. 86979

[12] McLaughlin K W, Yenen O, Jaecks D H, Gay T J, Sant'Anna M M, Calabrese D and Thaden-Jordan B 2002 Phys. Rev. Lett. 88 123003-1

[13] Minnhagen L 1963 Ark. Fys. 25203

Minnhagen L 1971 J. Opt. Soc. Am. 611257

[14] Statz H, Horrigan F A, Koozekanani S H, Tang C L and Koster G F 1965 J. Appl. Phys. 362278

[15] Smith N V, Hulbert S L, Johnson P D and Erskine J L 1994 Nucl. Instrum. Methods A 347119

Höchst H, Bulicke P, Nelson T and Middleton R 1995 Rev. Sci. Instrum. 661598

[16] Yenen O, McLaughlin K W, Jaecks D H and Snell G 2001 Proc. 22nd Int. Conf. on the Physics of Electronic and Atomic Collisions, Abstracts of Contributed Papers ed S Datz, M E Bannister, H F Krause, L H Saddiq, D Schultz and C R Vane (Princeton, NJ: Rinton) p 675

[17] Fano U and Macek J 1973 Rev. Mod. Phys. 45553

[18] Greene C H and Zare R N 1982 Ann. Rev. Phys. Chem. 33119

[19] Aymar M, Greene C H and Luc-Koenig E 1996 Rev. Mod. Phys. 681015

[20] Miecznik G, Greene C H and Robicheaux F 1995 Phys. Rev. A 51513

[21] van der Hart H W and Greene C H 1998 Phys. Rev. A 582097

[22] van der Hart H W and Greene C H 2002 Phys. Rev. A 65062509

[23] Gorczyca T W, Felfli Z, Zhou H-L and Manson S T 1998 Phys. Rev. A 583661

[24] van der Hart H W and Greene C H 1999 J. Phys. B: At. Mol. Opt. Phys. 324029

[25] Froese Fischer C, Brage T and Jönsson A 1997 Computational Atomic Structure: An MCHF Approach (Bristol: IOP Publishing)

[26] Kuhn H G 1969 Atomic Spectra 2nd edn (New York: Academic) pp 157-64

[27] Wijesundera W and Kelly H P 1989 Phys. Rev. A 39634

[28] Silfvast W T, Al-Salameh D Y and Wood O R II 1986 Phys. Rev. A 345164

[29] Moiseiwitsch B L and Smith S J 1968 Rev. Mod. Phy. 40238 
[30] Fano U 1985 Phys. Rev. A 32617

Fano U and Cooper J W 1968 Rev. Mod. Phys. 40441

[31] Cherepkov N A 1983 Adv. At. Mol. Phys. 19395

[32] Keßler J 1985 Polarized Electrons 2nd edn (Berlin: Springer) pp 144-56
[33] Smith K 1971 The Calculation of Atomic Collision Processes (New York: Wiley) pp 44-8

[34] Connerade J-P, Lane A M and Baig M A 1985 J. Phys. B: At. Mol. Phys. 183507

[35] Connerade J-P and Lane A M 1988 Rep. Prog. Phys. 511439 